\begin{tabular}{|c|c|c|}
\hline Beitr. Ent. & Keltern & ISSN 0005-805X \\
\hline $\mathbf{5 7}(2007) 2$ & S. $285-320$ & 21.12 .2007 \\
\hline
\end{tabular}

\title{
Tectusa montana aus den Alpen und die Tectusa-Arten der Karpaten - Tectusa-Studien 1
}

\section{(Coleoptera: Staphylinidae: Aleocharinae: Oxypodini)}

Mit 54 Figuren und 2 Verbreitungskarten

\section{LOTHAR Zerche}

\section{Zusammenfassung}

Tectusa montana (KraATZ, 1856) comb. n., ohne exakten Fundort beschrieben aus den Österreichischen Alpen, wird aus der Gattung Oxypoda neu kombiniert. Wegen ihrer Synonymisierung mit der lokalendemischen Parocyusa franzi Scheerpeltz, 1958 syn. n. (locus typicus: Österreich, Steiermark, Hochreichart) ist ihr locus typicus geklärt. Sie wird mit der verwandten Art Tectusa holdhausi (Bernhauer, 1902) verglichen. Die so genannte Oxypoda montana der meisten Autoren aus den Karpaten repräsentiert einen Komplex aus sechs geographisch getrennten Arten, die neu beschrieben werden: Tectusa transsylvanica sp. n. (locus typicus: Rumänien, Südkarpaten, Făgăraş, Bulea-See), Tectusa bucegiensis sp. n. (locus typicus: Rumänien, Südkarpaten, Bucegi, Omu-Massiv), Tectusa rosenauensis sp. n. (locus typicus: Rumänien, Südkarpaten, Rosenauer Gebirge $=$ Schuler $=$ Postavaru-Gipfel bei Poiana Braşov), Tectusa ceablauensis sp. n. (locus typicus: Rumänien, Ostkarpaten, Ceahlau-Massiv), Tectusa rodnaensis sp. n. (locus typicus: Rumänien, Nordostkarpaten, Rodnaer Gebirge $=$ Munții Rodnei) und Tectusa nigromontis sp. n. (locus typicus: Ukraine, Nordostkarpaten, Czerna Hora = Čhornohora). Für Oxypoda montana KraAtz, 1856 wird ein Lectotypus designiert. Eine Tabelle der sechs Karpaten-Arten wird gegeben. Die Verbreitung von zwei Arten aus den Alpen und der sechs Arten der Karpaten werden auf Karten abgebildet. Meldungen von "Oxypoda montana" aus der Hohen Tatra (Polen, Slowakei) sind weder durch Tiere noch durch exakte Funddaten belegt und deshalb äußerst zweifelhaft. Die Teile 1 (Hefte 1-2) und 2 (Hefte 3-4) der Publikation von KRAATZ (1856-1858) werden nach Sekundärquellen jeweils vordatiert.

\section{Summary}

Tectusa montana (KraAtz, 1856) comb. n., described from the Austrian Alps without an exact locality, is transferred from Oxypoda. The type locality is now known, because of its synonymization with the local endemic Parocyusa franzi Scheerpeltz, 1958 syn. n. (type locality: Austria, Steiermark, Hochreichart). It is compared with the related Tectusa holdhausi (Bernhauer, 1902). The so-called Oxypoda montana of most authors from the Carpathians represents a complex of six geographical separated species described as new: Tectusa transsylvanica sp. n. (type locality: Romania, South Carpathians, Făgăraş, Bulea lake), Tectusa bucegiensis sp. n. (type locality: Romania, South Carpathians, Bucegi, Omu massif), Tectusa rosenauensis sp. n. (type locality: Romania, South Carpathians, Rosenauer Gebirge $=$ Schuler $=$ Postavaru peak near Poiana Braşov), Tectusa ceahlauensis sp. n. (type locality: Romania, East Carpathians, Ceahlau massif), Tectusa rodnaensis sp. n. (type locality: Romania, Northeast Carpathians, Rodnaer Gebirge = Munții Rodnei), and Tectusa nigromontis sp. n. (type locality: Ukraine, North Carpathians, Czerna Hora = Čhornohora). For Oxypoda montana KraATZ, 1856 a lectotype is designated. A key is given for the six species of the Carpathians. The distribution of two species of the Alps and the six species from the Carpathians are mapped. Records of "Oxypoda montana" from the Vysoké Tatry (Poland, Slovakia) are neither substantiated by specimens nor by any exact collection date and therefore highly doubtful. Parts 1 (numbers 1-2) and 2 (numbers 3-4) of the publication by KRAATZ (1856-1858) are each allocated earlier dates using secondary sources. 


\section{Key words}

Coleoptera, Staphylinidae, Aleocharinae, Tectusa, new species, new synonym, new combination, key to Carpathian species, distribution, Austria, Poland, Romania, Slovakia, Ukraine.

\section{Neue Arten}

Tectusa transsylvanica sp. $\mathbf{n} .$, Tectusa bucegiensis sp. n., Tectusa rosenauensis sp. $\mathbf{n}$. , Tectusa ceablauensis sp. n., Tectusa rodnaensis sp. $\mathbf{n}$., Tectusa nigromontis sp. $\mathbf{n}$.

\section{Inhalt}

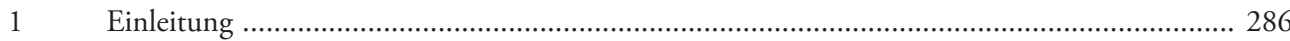

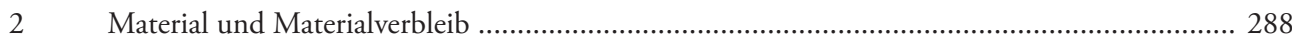

3 Tectusa montana (KraAtz, 1856) comb. n. - eine Art aus den Alpen ........................................ 288

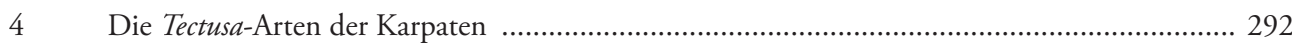

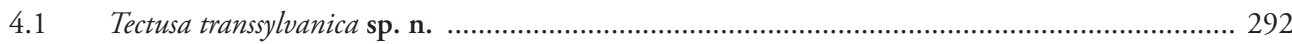

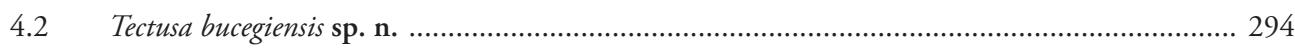

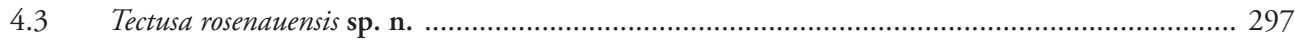

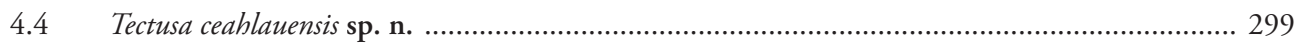

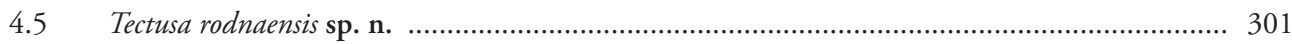

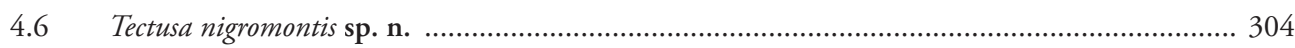

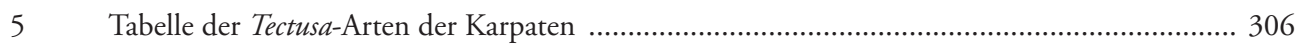

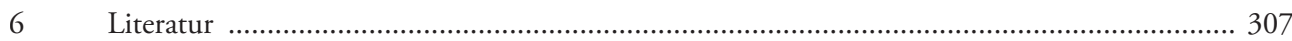

\section{Einleitung}

Oxypoda montana KraATZ wurde zuerst von ReITTER (1878) aus den Karpaten gemeldet, später von Ganglbauer (1895) mit den Karpaten in Zusammenhang gebracht, indem er schrieb "Österreich, Ungarn”. Auch Bernhauer (1902) hat in seiner Revision O. montana als eine Art der Karpaten aufgefasst: "Siebenbürgen. Von meinem Freunde Herrn Friedrich Deubel und anderen Sammlern in großer Zahl im Rodnaer Gebirge und auf dem Bucsecs gefangen. Oesterreichische Stücke habe ich nicht gesehen und halte ich das Vorkommen des Thieres bei uns für mehr als zweifelhaft." Die Art wird seither von allen Autoren, ausgenommen PORTA (1926), als in der subalpinen/alpinen Region der Karpaten weit verbreitet angesehen (z. B. Ganglbauer 1896, Holdihaus 1910, Deubel 1910, Petri 1912, Fenyes 1920, Winkler 1925, Roubal 1930, Horion 1967, Lohse 1974, Smetana 2004), aber auch für Mitteleuropa angeführt (Lohse 1974, BuraKowsKi \& al. 1981, BoHÁč 1993, Assing \& Schülke 2001, Smetana 2004, Assing \& Schülke 2007).

Die Untersuchung des Syntypus aus der Sammlung KraAtz, das Studium der Beschreibung und die Auswertung des "Verzeichniß der Käfer Deutschlands" (KraAtz 1869) brachten das überraschende Ergebnis, dass die KraATz'sche Art in Wirklichkeit aus Österreich stammt. Sie war von Reitter (1878), Ganglbauer (1895), Bernhauer (1902) und den späteren Autoren, ausgenommen PORTA (1926), fälschlich auf das karpatoendemische Taxon bezogen worden, weil sie eine solche Art aus Österreich nicht gekannt haben. Die falsche Deutung war so verfestigt, dass auch Horion (1967) die Angabe in der Originalbeschreibung als unrichtig angesehen hat: "Nicht im Alpengebiet; alte Angaben aus Österreich, die bis auf Kraatz 1858 [recte 1856] zurückreichen, und aus Norditalien (Porta 1926) sind unrichtig." Auch bezüglich Porta irrte Horion, denn dessen Angabe "Alpi" muss man nicht auf Norditalien beziehen. 
Das Taxon der Karpaten, das schon 1877 von Reitter und Leder und vor 1910 von Ganglbauer, Schubert, Holdhaus und vor allem Deubel, der mit vielen Koleopterologen in Tauschverbindung stand, in der Zeit zwischen den beiden Weltkriegen auch von tschechoslowakischen und polnischen Koleopterologen, häufig gesammelt wurde und in allen Sammlungen vorhanden ist, war durch die Verwechslung mit der Alpenart ohne einen Namen geblieben. Es hat sich bei der Untersuchung zusätzlich als ein Komplex geographisch getrennter Arten herausgestellt.

Die von den meisten Autoren bis heute so gröblich fehlgedeutete KraAtz'sche Art und die bis jetzt unter ihrem Namen fungierenden Arten der Karpaten aus sechs orogrophisch isolierten Massiven, die weder zur Gattung "Oxypoda” gehören noch etwas mit der Art "montana” zu tun haben, außer dass sie in die gleiche Gattung Tectusa Bernhauer, 1899 gehören, werden hiermit bekannt gemacht.

Möglicherweise existieren in den Karpaten noch mehr unerkannte Arten. Meldungen von „Oxypoda montana“ gibt es auch vom Csukás und vom Negoi (subalpin) (Deubel 1910, Horion 1967) sowie aus der Hohen Tatra (Lohse 1974, Smetana 2004), die hier nicht überprüft werden können. Von diesen Fundorten sind dem Autor keine Belege bekannt geworden.

Funde vom Negoi dürften zu T. transsylvanica sp. n. gehören, die aus dem gleichen Gebirge stammt.

Das Vorkommen in der Hohen Tatra erscheint dagegen äußerst zweifelhaft. Es gibt weder einen Beleg noch einen exakten Fundort. Roubal (1930) gibt die "Art" nur aus den Ostkarpaten an (“Cp. or.", “Čor. h.”), die heute zur Ukraine gehören. Horion (1967) kennt keinen Fund aus Mitteleuropa. Lohse (1974) nennt für seine Meldung aus der Tatra keine Quelle. Burakowski \& al. (1981) zitieren als Quelle für ihre Angabe "Tatry" nur LoHse (1974); aus Polen ist den Autoren aber kein Beleg bekannt. Auch die Angaben von Boháč (1993) für die Slowakei und die von Smetana (2004) für Polen und die Slowakei (Vysoké Tatry) sind ohne Quellenangabe.

Nicht geklärt werden zwei Tiere ( $\sigma^{\star}$, + ) mit der folgenden Etikettierung, weil ihre Herkunft unklar ist und die Spermatheka des Weibchens (Fig. 42) deutlich von Spermatheken aus dem Făgăraş-Massiv abweicht: “Transsylv. Alpen, R. Turm-Paß, 1917, Dr. Maertens” (MNHUB). Bei der Fundortangabe Roter-Turm-Pass [= Pas Turnu Roşu] kann der nächste subalpine/alpine Gipfel gemeint sein, entweder östlich in den Munții Făgăraşului oder auch westlich des Passes. In beiden Richtungen sind solche Gipfel nur wenige Kilometer entfernt. Der Pass selbst ist mit $354 \mathrm{~m}$ NN für ein Vorkommen von Tectusa viel zu niedrig. ${ }^{1}$ Wenn der Fundort aber exakt angegeben wurde, muss man vom Anschwemmen durch Hochwasser ausgehen, wahrscheinlich aus einem benachbarten Massiv. Bei Hochwasser ist aber auch ein Ferntransport über den Alt (Olt) nicht völlig auszuschließen.

Aus den vom Roter-Turm-Pass noch über etwa $140 \mathrm{~km}$ nach Westen reichenden ausgedehnten subalpinen/alpinen Massiven sind bisher noch keine Tectusa bekannt geworden ${ }^{2}$ (Karte 2, S. 295). Auch eine Sammelexkursion des Autors in das Gebiet des Netiş-Sees und des Bloju-Gipfels in den Munții Tarcului im Juli 1985 erbrachte keine Tectusa.

\section{Danksagung}

Lutz Behne (DEI) sammelte viermal mit mir gemeinsam in den Karpaten. Gemeinsam mit Lutz Behne und Manfred Kahlen, Hall i. T., wurde am Hochreichart Tectusa franzi gesammelt, die sich später als synonym mit Tectusa montana erwiesen hat.

\footnotetext{
${ }^{1}$ Mit den gleichen Fundortetiketten gibt es auch die alpine Art Hypsonothrus deubeli (Zerche 1990). Das Sammeln auf einem benachbarten Gipfel ist deshalb wahrscheinlicher.

${ }^{2}$ Es gibt aus diesem Gebiet auch keine Coryphiini (Staphylinidae: Omaliinae) (Zerche 1990, 1993).
} 
Für die Ausleihe von Material, teilweise schon vor langer Zeit, danke ich Volker Assing, Hannover, Didier Drugmand, Institut royal des Sciences naturelles de Belgique, Bruxelles, Vladimir Janskú, Slovenské Národné Múzeum Bratislava, Josef JelineK, Narodni Muzeum v Praze, Prag, Manfred Kahlen, Tiroler Landesmuseum Ferdinandeum Innsbruck, Damir Kovac und Andrea Hastenpflug-Vesmanis, Forschungsinstitut Senckenberg, Frankfurt am Main, Rüdiger Krause und Olaf Jaeger, Staatliches Museum für Tierkunde Dresden, Mieczyslaw Mazur, Institute of Systematics and Evolution on Animals, Polish Academy of Sciences, Kraków, Paul Mildner, Landesmuseum Kärnten, Klagenfurt, Alfred Newton and James Boone, Field Museum of Natural History Chicago, Jan RŮžžčKa, Prag, Wolfgang Schawaller, Staatliches Museum für Naturkunde Stuttgart, Harald Schillhammer, Naturhistorisches Museum Wien, Manfred Uhlig, Johannes Frisch und Joachim Willers, Museum für Naturkunde der Humboldt-Universität, Berlin, und Paul Wunderle, Mönchengladbach. Dieter Simon, Universitatea "Transsilvanica", Facultatea de Silvicultura, Braşov, danke ich für die Möglichkeit, in der Sammlung Deubel zu arbeiten.

Derex Lott, Loughborough, kontrollierte die englische Zusammenfassung. Die Habitusfotos und die Kartenvorlagen fertigte Lutz Behne, die Zeichnungen in Tusche verdanke ich Ute Kunter (beide DEI). Lutz BeHNe danke ich auch für vielfache andere technische Hilfen.

\section{Material und Materialverbleib}

Für diese Studie wurden 941 Individuen (T. montana 35, T. holdhausi 30, Tectusa-Arten der Karpaten 876) aus 17 Sammlungen untersucht. Die Holotypen der neuen Arten befinden sich im im CNHM (1) und im DEI (5).

$\begin{array}{ll}\text { cAs } & \text { coll. Assing, Hannover, } \\ \text { CNHM } & \text { Field Museum of Natural History, Chicago, } \\ \text { cRǓ } & \text { coll. RǔŽıčKa, Prag, } \\ \text { cWU } & \text { coll. WundERLE, Mönchengladbach, } \\ \text { DEI } & \text { Deutsches Entomologisches Institut, Müncheberg, } \\ \text { FIS } & \text { Forschungsinstitut und Museum Senckenberg, Frankfurt am Main, } \\ \text { IRSNB } & \text { Institut royal des Sciences naturelles de Belgique, Bruxelles, } \\ \text { LMKK } & \text { Landesmuseum Kärnten, Klagenfurt, } \\ \text { MNHUB } & \text { Museum für Naturkunde der Humboldt-Universität, Berlin, } \\ \text { MTD } & \text { Staatliches Museum für Tierkunde Dresden, } \\ \text { NHMW } & \text { Naturhistorisches Museum Wien, } \\ \text { NMP } & \text { Narodni Muzeum v Praze (Prag), } \\ \text { PANK } & \text { Institute of Systematics and Evolution on Animals, Polish Academy of Sciences, Kraków, } \\ \text { SMNS } & \text { Staatliches Museum für Naturkunde Stuttgart, } \\ \text { SNMB } & \text { Slovenské Národné Múzeum Bratislava, } \\ \text { TLFI } & \text { Tiroler Landesmuseum Ferdinandeum Innsbruck, } \\ \text { UTFS } & \text { Universitatea “Transsilvanica”, Facultatea de Silvicultura, Braşov. }\end{array}$

\section{Tectusa montana (KraAtz, 1856) comb. n. - eine Art aus den Alpen}

Fig. 1-3, 13-15, Karte 1

Oxypoda montana Kratz, 1856: 187; Redtenbacher 1856: 145; Gemminger \& Harold 1868: 528; Kratz, 1869: 12; Redtenbacher 1872: 153; Porta 1926: 203, nec Reitter 1878: 38, Ganglbauer 1895: 76, BERnHAUER 1902: 190 [104] et auctorum. 
Parocyusa franzi Scheerpeltz, 1958: 109 syn. n.; Horion 1967: 297; Lohse 1989: 226.

Chilopora (Parocyusa) franzi; LoHSE 1974: 242.

Tectusa franzi; AssING \& SCHÜLKe 2007: 75.

\section{Typenmaterial}

\section{Oxypoda montana}

Lectotypus ( $\sigma^{\text {( ) }}$ : alp / montana mihi [KraATZ script., Sammlungsetikett] / coll. Kraatz [gedruckt] / LECTOTYPUS Oxypoda montana Kraatz, Zerche desg. 2007 / Tectusa montana (Kraatz, 1856), det. Zerche 2007 (Fig. 1) (DEI). Hiermit designiert!

Anmerkung: KRaATZ lag wahrscheinlich nur dieses eine Tier vor, er hat aber die Anzahl seiner Typen nicht angegeben. Der Lectotypus ist etwas beschädigt. Es fehlen rechts die Antennenglieder $\mathrm{V}$ bis XI und das Mittelbein (Fig. 2).

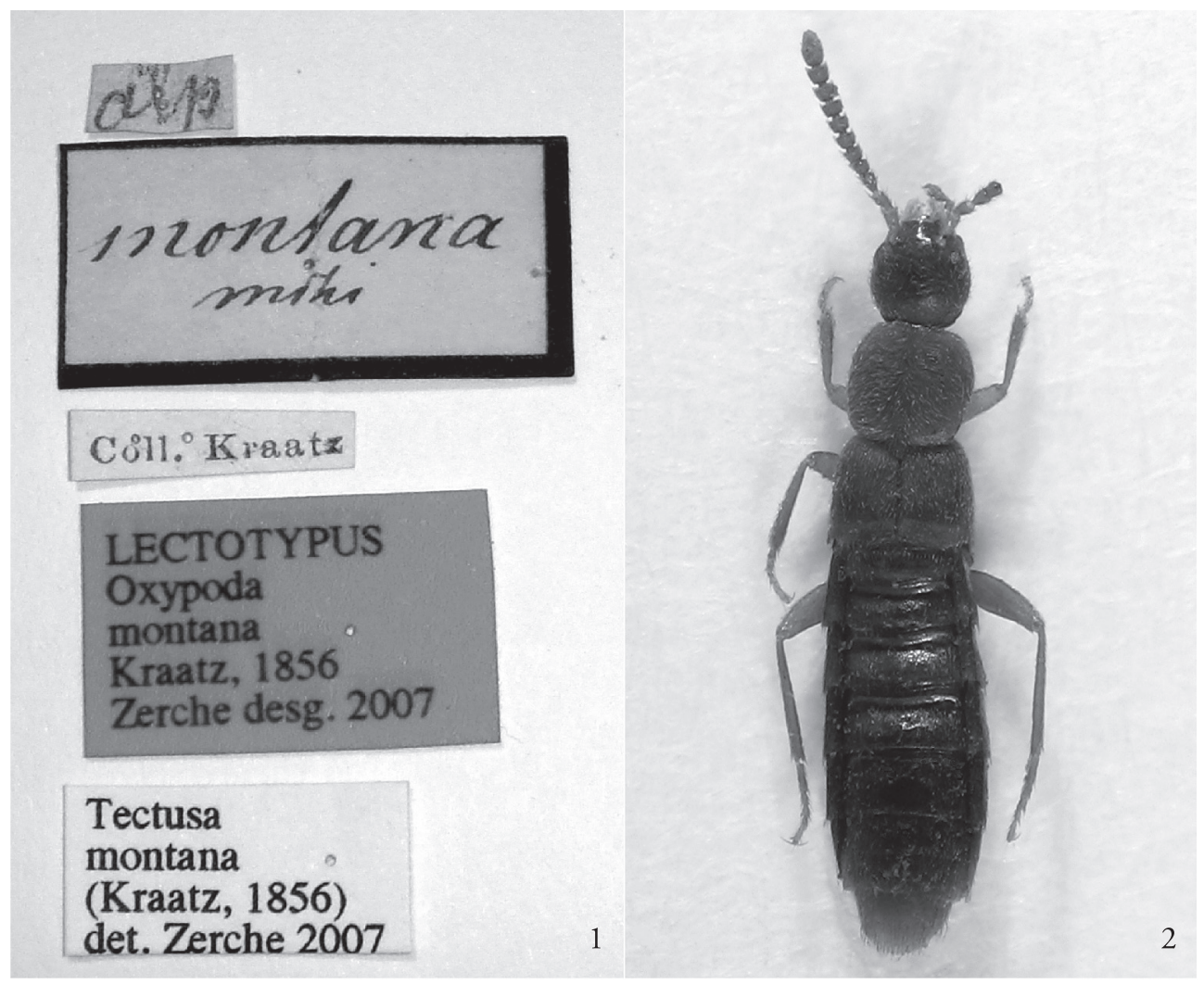

Fig. 1-2: Tectusa montana (KraAtz). - 1 Etiketten des Lectotypus. 2 Habitus [0َ, Lectotypus].

\section{Parocyusa franzi}

Der Holotypus ist verschollen! In der Sammlung Scheerpeltz (NHMW) steckt nur das leere Aufklebeplättchen. Der Holotypus wurde vergeblich in der Schachtel gesucht und muss als verloren gelten (22.08.1995, SCHILlHAMmer i. 1.). 


\section{Anmerkungen zum locus typicus}

In der Beschreibung gibt KraATZ an: „In den gebirgigen Theilen Österreichs, äußerst selten“. Auf dem Etikett des Tieres steht dagegen nur in Bleistiftschrift "alp" [alpin] (Fig. 1). KraAtz hat das Tier offensichtlich aus dritter Hand bekommen, seine genaue Herkunft nicht gekannt und den Sammler nicht gewusst, denn an anderer Stelle hat er bei Seltenheiten den Sammler genannt.

Dass die Verlegung des locus typicus in die Karpaten durch Bernhauer (1902) falsch ist, ergibt sich auch aus dem Serientitel des Buches. In die "Naturgeschichte der Insecten Deutschlands" hat KraAtz (1856-1858) nur Arten aus dem damaligen Deutschland aufgenommen, und Österreich war Mitglied im Deutschen Bund, Ungarn jedoch nicht.

Durch die Synonymisierung mit der lokalendemischen Art Parocyusa franzi ist der locus typicus geklärt: Nordsteiermark, Seckauer Alpen, Hochreichart [Gipfelhöhe 2.416 m; auch Hochreichhart oder Hochreichard, die Landkarten sind nicht einheitlich; ca. $47^{\circ} 20^{\prime} \mathrm{N}, 14^{\circ} 42^{\prime} \mathrm{O}$ ].

\section{Beschreibung}

Maße des Lectotypus [in mm]: Körperlänge 3,12; Kopfbreite 0,46; Antennenlänge 1,06; Augenlänge 0,11 ; Schläfenlänge 0,23; Pronotumlänge 0,52; Pronotumbreite 0,58; Nahtlänge 0,35; Elytrenbreite 0,64; Abdomenbreite 0,70; Glied I der Hintertarsen 0,14; Glieder II bis IV der Hintertarsen zusammen 0,16; Aedoeaguslänge 0,48.

Körper zweifarbig: Kopf düster rotbraun; Mitte des Tergits IV und die Tergite V bis VII mit Ausnahme ihrer Hinterränder geschwärzt. Pronotum, Scutellum, Elytren, Abdomenspitze, Mundteile und Antennenglieder III bis XI hell rotbraun. Scapus, Pedicellus und Beine gelbrot. Oberseite dicht und deutlich chagriniert, schwach glänzend, die Elytren geringfügig stärker glänzend. Punktur fein und dicht, aber undeutlich; nur auf den Elytren etwas stärker ausgebildet und deutlicher. Behaarung fein und anliegend; auf dem Kopf schräg nach vorn und innen gerichtet; auf dem Pronotum vom Typ III (nach BeNICK \& LoHSE 1974: im vorderen Fünftel der Mittellinie nach vorn gerichtet, in den übrigen vier Fünfteln nach hinten gerichtet); auf den Elytren annähernd gerade nach hinten gerichtet.

Kopf groß, aber merklich schmaler als das Pronotum (0,79); nach hinten erweitert, über den gerundeten Schläfen am breitesten. Augen etwas aus der Kopfrundung vorgewölbt, klein, viel kürzer als die Schläfen $(0,48)$. Stirnnaht gerade, fein, aber gut erkennbar. Schläfenrandung vollständig.

Antennen relativ kräftig und gestreckt, zurückgelegt etwa die Mitte der Elytren erreichend. Maße der Antennenglieder [in $\mu \mathrm{m}$ ]: I: 136 x 64; II: 112 x 48; III: $112 \times$ 64; IV: $60 \times$ 74; V: $72 \times$ 82; VI: 75 x 88; VII: 75 x 88; VIII: 72 x 96; IX: 80 x 101; X: 80 x 90 XI: 176 x 104.

Maxillarpalpen schlank, Glied III deutlich länger als der Pedicellus der Antennen $(1,29)$; Maße des Glieds III [in $\mu \mathrm{m}]: 144$ x $51(2,82)$.

Pronotum etwas quer $(1,12)$, deutlich breiter als der $\operatorname{Kopf}(1,26)$, schmaler als die Elytren $(0,91)$; knapp vor der Mitte am breitesten; nach vorn kaum stärker als nach hinten verengt. Seitenränder gleichmäßig stark gebogen, ohne Ausschweifung. Vorderrand konvex. Hinterrand gerade. Hinterwinkel stumpf, aber klar erkennbar. Fläche mäßig gewölbt, mit sehr flacher, aber deutlicher Mittellängsfurche. Hypomeren wie bei allen Tectusa-Arten bei Ansicht im Profil sichtbar.

Elytren flach gewölbt, Naht in den vorderen drei Vierteln furchenartig vertieft; über den kaum abgeschrägten Schultern nicht schmaler als das Pronotum, nach hinten fast geradlinig erweitert.

Naht etwas mehr als halb so lang wie die größte Breite $(0,55)$. Hinterrand zur Naht stumpfwinklig eingezogen, neben den Hinterwinkeln etwa in gleicher Tiefe eingebuchtet. 
Abdomen kaum erweitert, über dem Segment V am breitesten, annähernd parallel. Tergite III bis V mit mäßig tiefem, Tergit VI mit flachem Basaleindruck. Tergit VII wenig länger als Tergit VI $(1,20)$, ohne Fransensaum.

Glied I der Hintertarsen ziemlich gestreckt, etwas länger als die Glieder II und III zusammen $(1,10)$.

$0^{\star}$ : Antennenglied XI im Apikaldrittel abgeschnürt. Hinterrand des Sternits VIII parabelförmig. Aedoeagus ohne paarige ventrale Medianfortsätze (Fig. 13-14). Apikalfortsatz der Paramere (Fig. 15).

ㅇ: Spermatheka (Fig. 16).

\section{Differentialdiagnose}

Tectusa montana ähnelt unter den Arten der Alpen am meisten T. holdhausi (BERnHAUER, 1902), die ihr mit $95 \mathrm{~km}$ Entfernung auch räumlich am nächsten ist. Tectusa holdhausi ist bisher nur aus dem südöstlichen Kärnten bekannt und besiedelt dort ein kleines Areal, das sich aber über mehrere benachbarte Bergmassive in den Karawanken (Hochobir, Koschuta, Petzen) und Steiner Alpen (Vellacher Kotschna: Sanntaler und Seeländer Sattel) erstreckt (Karte 1, siehe auch NeuHÄUSERHAPPE 1999). Tectusa holdhausi ist weniger kontrastreich gefärbt, nur undeutlich zweifarbig. Ihr Pronotum ist schmaler und viel stärker gewölbt (Fig. 2-3, 4). Ihr Antennenglied XI ist in beiden Geschlechtern schlanker; bei den $0^{\star} o^{\star}$ sind die Unterschiede auffällig. Der Aedoeagus von T. holdhausi weicht im Innenbau und in der äußeren Form merklich ab (Fig. 14, 17); er ist aber auch deutlich länger (113\%) und vor allem breiter (122\%). Auch die Apikalfortsätze der Parameren (Fig. 15, 18) und die Spermatheken (Fig. 16, 19) der beiden Arten sind recht unterschiedlich.

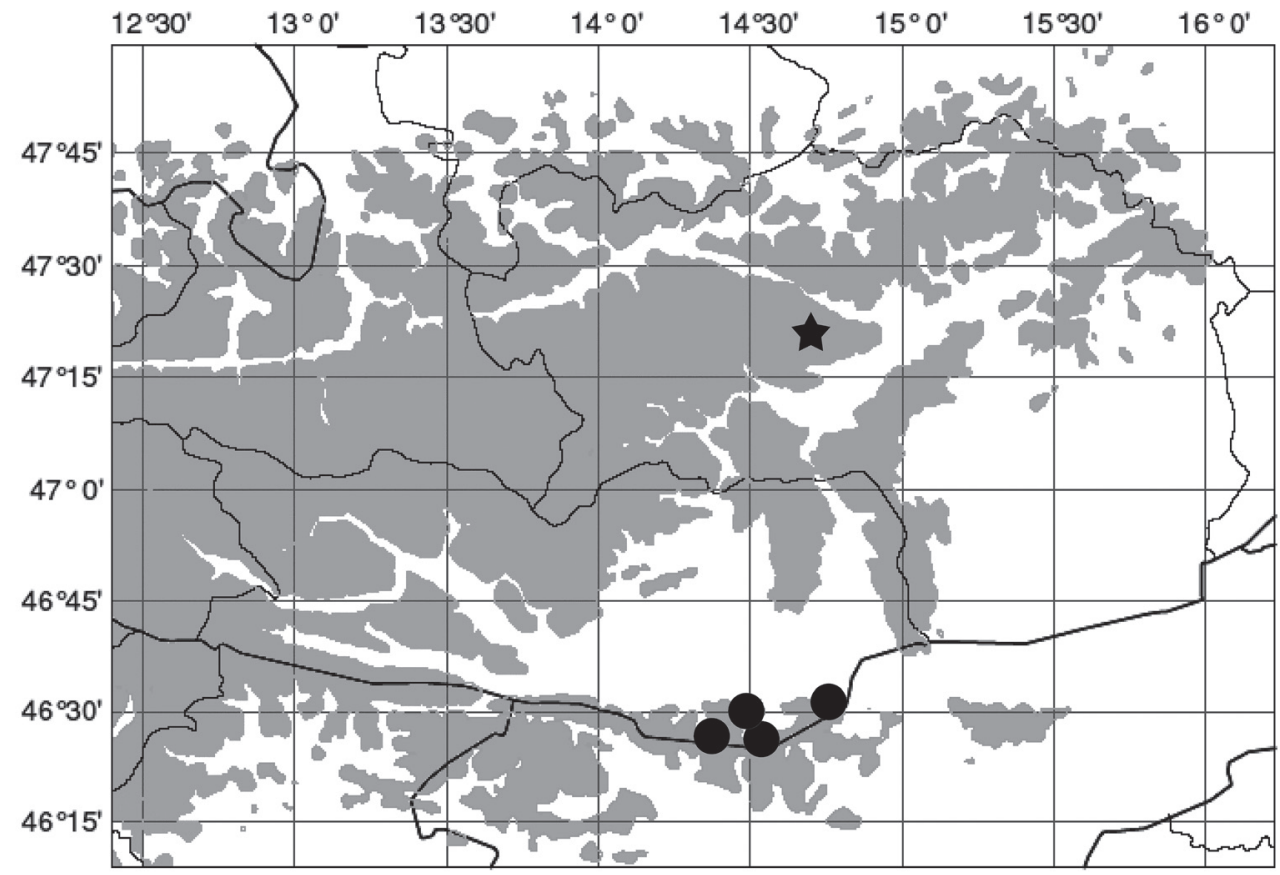

Karte 1: Verbreitung von Tectusa montana (KRAATZ) [Stern] in der Steiermark und von T. holdhausi (Bernhauer) [Kreis] in Kärnten. Karte aus Diepenbroek \& al. (2000). 
Von den sechs Tectusa-Arten der Karpaten ist T. montana wegen ihrer schlankeren Körperform, insbesondere durch ihr weniger queres Pronotum, und helleren Farbe auf den ersten Blick zu unterscheiden (vergleiche Fig. 2-3 mit Fig. 5-10). Tectusa montana fehlt das abgeleitete Merkmal "paarige mediane Fortsätze des Aedoeagus" der Arten der Karpaten, so dass sie auch nicht eng mit diesen verwandt ist. Ein gemeinsames Vorkommen kann man ausschließen; es handelt sich jeweils um lokale Endemiten.

\section{Verbreitung}

Die Art ist bisher nur vom Hochreichart und dem etwa $1 \mathrm{~km}$ entfernten, südöstlich angrenzenden Brandstetter Törl, dem Pass zwischen den Gipfeln Hochreichart und Brandstetterkogel, sowie von dem etwa 1,1 km entfernten, nördlich angrenzenden Schönebentörl bekannt (Karte 1). Sie ist in den Seckauer Alpen endemisch.

\section{Bionomie}

Es sind nur die Sammelumstände bekannt. Die meisten Exemplare wurden aus lockeren Rasen am Schneerand gesiebt, einige auch aus der Streu von Pinus mugo und Rhododendron ferrugineum.

\section{Untersuchtes Material}

A: Steiermark, Seckauer Alpen, Brandstettertörl östl. Hochreichhart, 2.100 m, Schneefeldrand, 17.06.1995, 2 o $^{\star}$, leg. Zerche (DEI); A: Steiermark, Seckauer Alpen, Hochreichhart, O-Seite, 2.200 m, Schneefeldrand, 17.06.1995, 1 ơ, leg. Zerche (DEI); A: St., Seckauer Alpen, Hochreichhart, leg. Kahlen / SO-Hang, 2.250 m, 17.6.1995, Festuca-Rasen, an Schneerand, 2 우 우 (TLFI; DEI); A: St., Seckauer Alpen, Hochreichhart, leg. Kahlen / O-Hang, 2.250 m, 17.6.1995, Rasen am Schneerand, 3 ơ o (TLFI); A: St., Seckauer Alpen, Brandstedtertörl, leg. Kahlen / 2.000 m, 17.6.1995, Latschenstreu, 1 (TLFI); A-St., Seckauer Alpen, Schönebentörl, leg. Kahlen / 2050 m, 5.7.2001, Rhododendronstreu zwischen Blöcken, 1 ơ, 3 우 ㅇ (TLFI); A-St., Seckauer Alpen, Hochreichhart Gipfel, leg. Kahlen / 2400-2416 m, 5.7.2001, Festuca-Rasen in Blockhalde unter Stein, $5 \sigma^{\star} \sigma^{\star} 4$ ㅇ ㅇ (DEI, TLFI); A-St., Seckauer Alpen, Hochreichhart ESeite, leg. Kahlen / 2100-2200 m, 4.7.2001, Rasen im Schutt an Schneerand, 5 ơ o đa $^{7}$ 우 우 (DEI, TLFI).

Untersuchte Exemplare: $180^{\star} \diamond^{\star}, 17$ 우 우.

\section{Die Tectusa-Arten der Karpaten}

\subsection{Tectusa transsylvanica sp. $\mathrm{n}$.}

Fig. 5, 11, 20-25, Karte 2

Oxypoda montana; Ganglbauer 1895: 76; Bernhauer 1902: 190 [104] et auctorum nec KraAtz, 1856: 187 , ex parte.

\section{Typenmaterial}

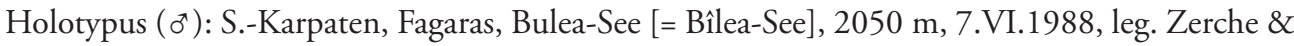
Behne / HOLOTYPUS Tectusa transsylvanica Zerche (DEI).

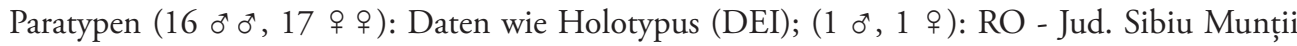


Făgăraşului leg. Kahlen / Vîrful Vînatoărea lui Buteanu 2506 m 18.7.1998 Dryasrasen (TLFI); (5 $0^{\star} o^{\star}, 2$ 9 +, 4 Ex.): RO - Jud. Sibiu Munții Făgăraşului leg. Kahlen / Oberer Bîlea-Kessel 2100 m 17.7.1998 Saxifraga aizoid.-Rasen (TLFI).

\section{Beschreibung}

Maße des Holotypus [in mm]: Körperlänge 3,60; Kopfbreite 0,53; Antennenlänge 1,18; Augenlänge 0,12 ; Schläfenlänge 0,21 ; Pronotumlänge 0,56 ; Pronotumbreite 0,71 ; Nahtlänge 0,32 ; Elytrenbreite 0,75; Abdomenbreite 0,79; Glied I der Hintertarsen 0,19; Glieder II bis IV der Hintertarsen zusammen 0,17; Aedoeaguslänge 0,66.

Farbe im ausgereiften Zustand düster rotbraun. Kopf und Abdomen meist pechbraun, dunkler als der übrige Körper. Maxillarpalpen angedunkelt. Basis der Antennen und Beine heller rotbraun. Abdomenspitze etwas aufgehellt. Oberseite dicht chagriniert, nur matt glänzend. Punktur fein, im Chagrin schwer sichtbar, auf Pronotum und Elytren viel deutlicher als auf dem Kopf und etwa von gleicher Stärke. Behaarung sehr fein und anliegend, auf den Elytren fast gerade nach hinten gerichtet.

Körperlänge: 3,05-4,20 mm (MW 3,51; $\mathrm{n}=15$ ).

Kopf groß, aber deutlich schmaler als das Pronotum $(0,75)$; nach hinten gerundet erweitert, über den Schläfen am breitesten. Augen flach, aber etwas aus der Kopfrundung vorgewölbt, im Profil etwas mehr als die Hälfte der Schläfenlänge messend (0,57). Stirnnaht gerade. Schläfenrandung vollständig.

Antennen relativ schlank, zurückgelegt die Mitte der Elytren etwas übertreffend. Maße der Antennenglieder ( $\left.o^{\top}\right)$ [in $\mu \mathrm{m}$ ]: I: 128 x 63; II: 120 x 58; III: 120 x 64; IV: 80 x 64; V: 80 x 72; VI: 77 x 80; VII: 80 x 85; VIII: 80 x 85; IX: 83 x 93; X: 88 x 93; XI: 160 x 88 .

Maxillarpalpen ziemlich gestreckt; Maße des Glieds III [in $\mu \mathrm{m}$ ]: 160 x 48.

Pronotum deutlich quer $(1,27)$, breiter als der $\operatorname{Kopf}(1,34)$, kaum schmaler als die Elytren $(0,95)$; etwa in der Mitte am breitesten, nach vorn kaum stärker als nach hinten verengt. Vorder- und Hinterwinkel breit abgerundet. Vorderrand konvex, Hinterrand annähernd gerade. Fläche stärker gewölbt, mit einer \pm deutlichen Abflachung oder Andeutung einer Vertiefung in der Mitte. Hypomeren bei Ansicht im Profil sichtbar.

Elytren flach gewölbt, über den abgeschrägten Schultern etwas schmaler als das Pronotum, nach hinten fast geradlinig erweitert. Naht etwas vertieft, viel kürzer als die größte Breite $(0,43)$. Hinterrand zur Naht stumpfwinklig eingezogen, neben den Hinterwinkeln etwa in gleicher Tiefe eingebuchtet.

Abdomen kaum erweitert, über dem Segment V etwas breiter als die Elytren $(1,05)$. Tergite III bis V mit tiefen Basaleindrücken, Tergit VI mit deutlich schwächerem Basaleindruck. Tergit VII deutlich länger als Tergit VI (ca. 1,25), ohne Fransensaum.

Glied I der Hintertarsen gestreckt, etwas länger als die Glieder II bis IV zusammen.

$o^{\star}$ : Aedoeagus (Fig. 20-21). Paarige ventrale Fortsätze (Fig. 22). Apikalfortsatz der Paramere (Fig. 23).

† : Körper durchschnittlich etwas größer und breiter. Spermatheka (Fig. 24-25).

\section{Differentialdiagnose}

Tectusa transsylvanica sp. n. wird mit den beiden anderen Arten aus den Südkarpaten verglichen, mit denen sie nicht gemeinsam vorkommt. Sie ist etwas breiter gebaut als T. bucegiensis $\mathbf{s p .} \mathbf{n}$. vom Bucegi-Massiv und T. rosenauensis sp. n. vom Gipfel des Schuler. Ihr Pronotum ist etwas 
schwächer gewölbt, die Schultern der Elytren sind etwas weniger abgeschrägt und nach hinten schwächer erweitert als bei diesen beiden Arten.

\section{Bionomie}

Es sind nur die Sammelumstände bekannt. Der Holotypus und 33 Paratypen wurden zwischen Felsen und grobem Geröll aus alpinen Polsterpflanzen gesiebt, an den wenigen vom Wind schneefrei gefegten Stellen oberhalb des Kessels (Fig. 11). Der tiefer liegende Kessel um den Bulea-See war unter einer tiefen Schneedecke verborgen. Begleitarten waren Hypsonothrus deubeli (Ganglbauer), Stenus zerchei Puthz und Dichotrachelus kimakowiczi Flach. Kahlen sammelte die Art aus Dryas octopetala-Rasen und aus Saxifraga aizoides-Rasen. Die Höhenangaben reichen von $2.050-2.506 \mathrm{~m}$.

\section{Verbreitung}

Die Art ist bisher nur aus dem Fagaras-Massiv [= Munții Făgăraşului] bekannt und hier wahrscheinlich endemisch (Karte 2).

Untersuchte Exemplare: $23 \sigma^{\star} \sigma^{\star}, 21$ 우 우, 4 Ex.

Derivatio nominis: Der Name transsylvanica (Adjektiv) nimmt auf den locus typicus in den Transsylvanischen Alpen in den Süd-Karpaten Bezug.

\subsection{Tectusa bucegiensis sp. $\mathrm{n}$.}

Fig. 6, 12, 26-31, Karte 2

Oxypoda montana; Ganglbauer 1895: 76; Bernhauer 1902: 190 [104] et auctorum nec Kratz, 1856: 187, ex parte.

\section{Typenmaterial}

Holotypus ( $\left.\sigma^{\star}\right)$ : Rum. S.-Karpaten, Bucegi-Geb., Omul [recte Omu], 2300 m, 19.VI.1987 / Schneefeldrand leg. Zerche \& Behne / HOLOTYPUS Tectusa bucegiensis Zerche (DEI).

Paratypen (10 $\sigma^{\star} o^{-}, 4$ ㅇ ㅇ, 74 Ex.): Daten wie Holotypus (DEI); (2 Ex.): Daten wie Holotypus (coll. Lohse: MHNG); (1 o*, 1 \%): Daten wie Holotypus (coll. Assing); (2 Ex.): Daten wie Holotypus (coll. Wunderle); (2 Ex.): Daten wie Holotypus (coll. Ulbrich: SMNS); (4 $\sigma^{\star} \sigma^{\star}$, 3 우 우, 15 Ex.): Daten wie Holotypus, aber 2100m (DEI); (1 $\sigma^{*}, 2$ 우 ㅇ): Rum. S.-Karpaten, Bucegi-Geb., Hütte Malaesti, 1800 m / 20.VI.1987, leg. Zerche \& Behne (DEI); (25 o o o 2 ㅇ 우 12 Ex.): Rum. S.-Karpaten, Bucegi-Geb., Umg. Babele, 2300 m / 18.VI.1987, leg. Zerche \& Behne (DEI); (20 o o , 5 우 우, 9 Ex.): Rum. Bucégi Geb., Umg. Babele, 2200 m, 20.VI.1986, leg. Zerche \& Behne (DEI); (2 $0^{\star} o^{\star}, 2$ ㅇ ㅇ , 30 Ex.): Rum. Bucégi Geb., Umg. Bucsoi, 2200 m / 21.VI.1986, leg. Zerche \& Behne (DEI); (2 Ex.): Ganglb. 95, Bucsecs Tr. (SMNS); (1 ơ , 4 ㅇ ㅇ): Transsylvania, Bucsecs, Leonhard (DEI); (1 \%): Ganglb. 95, Bucsecs Tr. / Oxypoda montana Kr. (PANK); (2 Ex.): Ganglb. 95, Bucsecs Tr. (coll. Koltze: DEI); (3 Ex.): Ganglb. 95, Bucsecs Tr. / Coll. Linke, Leipzig, Geschenk 1979 (MTD); (2 Ex.): Ganglb. 95, Bucsecs Tr. / montana Kr. / ex coll. Scheerpeltz (NHMW); (1 o o $^{*}$ : Tr: Bučeč, VII.1912, Obenberger / montana Roubal det. (SNMB: Nicht als Paratypus etikettiert!); (1 Ex.): Bučeč, Transsyl. (SNMB: Nicht als Paratypus etikettiert!); (2 $\sigma^{\star} \sigma^{\star}, 1$ Ex.): Bucsecs, Deubel / alpin / montana / Paganetti (coll. HeYden: DEI); (1 Ex.): Bucsecs, Deubel / Oxypoda montana Kr. / CNHM 1955 Eduard Knirsch Palaearctic Colln. (CNHM); (7 Ex.): Bucsecs, Deubel / alpin / montana Kr. det. Bernhauer / Chicago NHMus M. Bernhauer Collection (CNHM); (1 Ex.): Bucsecs, Deubel / Oxypoda montana Kr. / CNHM 


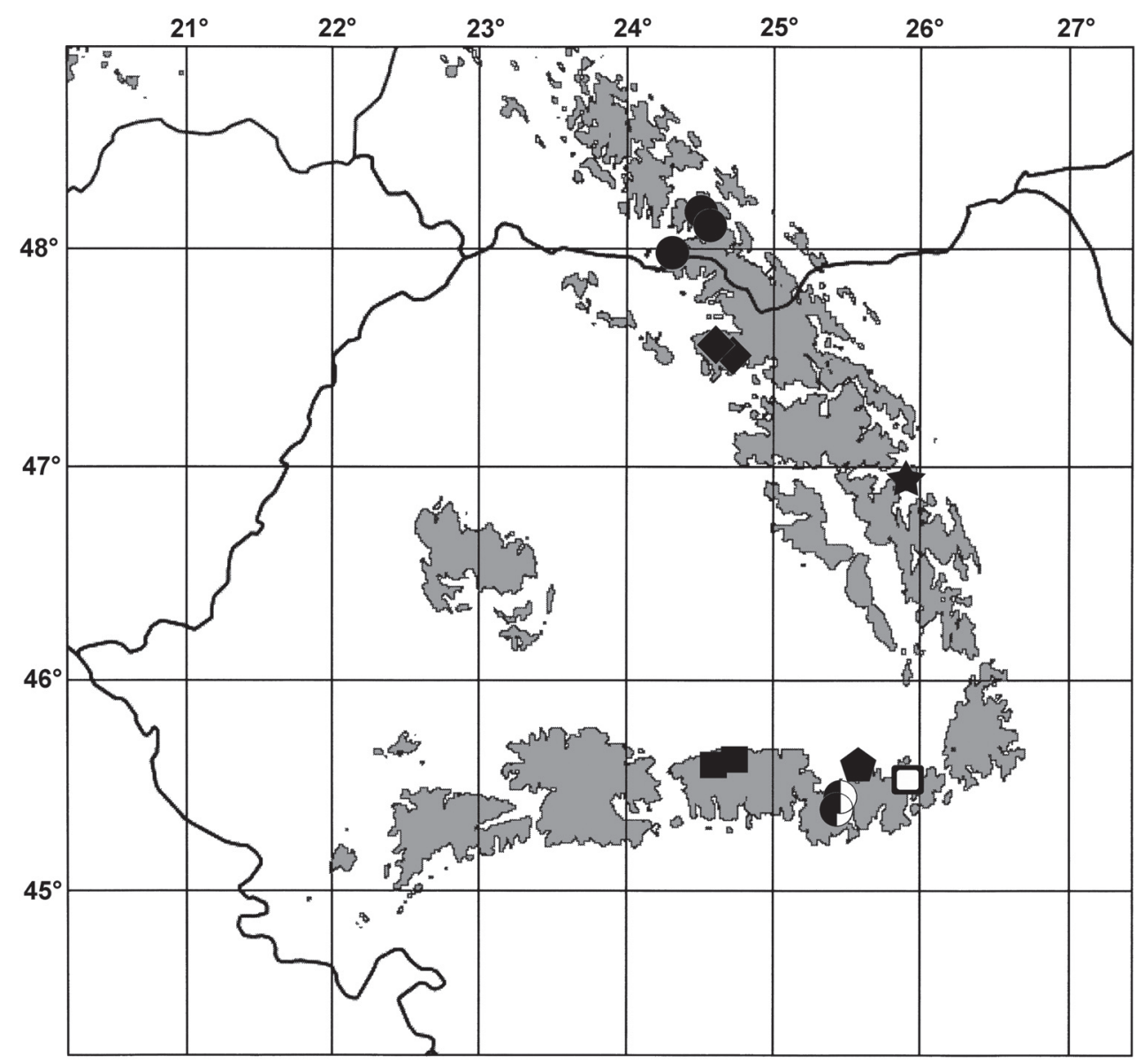

Karte 2: Verbreitung von Tectusa nigromontis sp. n. [geschlossener Kreis] in der Ukraine und von T. rodnaensis sp. n. [Quadrat auf Spitze], T. ceablauensis sp. n. [Stern], T. rosenauensis sp. n. [Fünfeck], T. bucegiensis sp. $\mathbf{n}$. [halbgeschlossener Kreis], T. transsylvanica sp. n. [Quadrat auf Seite] und Tectusa spec. [offenes Quadrat; Ciucaş (Deubel 1910)] in Rumänien. Karte aus Diepenbroek \& al. (2000).

1955 Eduard Knirsch Palaearctic Colln. (CNHM); (1 ơ, 2 Ex.): Ganglb. 95, Bucsecs Tr. / montana Kr. [Bernhauer script.] / Chicago NHMus M. Bernhauer Collection (CNHM); (1 o'): Ganglb. 95, Bucsecs Tr. / montana Kr. Siebenbürgen Ganglbauer [Bernhauer script.] / Chicago NHMus M. Bernhauer Collection (CNHM); (1 Ex.): Ganglb. 95, Bucsecs Tr. / Oxypoda montana Kr. / CNHM 1955 Eduard Knirsch Palaearctic Colln. (CNHM); (1 Ex.): Buczecz Tr., alpin / CNMH 1955 Karl Brancsik Colln. ex Eduard Knirsch (CNHM); (1 o*, 1 Ex.): Bucsecs 98, Schuster / montana Kr. Siebenbürgen [Bernhauer script.] / Chicago NHMus M. Bernhauer Collection (CNHM); (4 Ex.): Bucsecs 98, Schuster / Bernh. det. / montana / coll. Schuster (NHMW); (1 Ex.): wie vorige, aber zusätzlich S. O. Karpathen Bucsecs (NHMW); (7 o o $\sigma^{\star}, 11$ Ex.): Bucsecs 98, Schuster / montana Kr. / coll. Klima (NHMW); (2 o $\left.^{\star} o^{\star}\right)$ : Bucsecs, Deubel / $\sigma^{\star} /$ alpin / montana Kr. / ex coll. Scheerpeltz (NHMW); (55 Ex.): Buczecz Tr., subalpin / Oxypoda montana (NMP); (1 Ex.): Deubel, Bucsecs / Coll. Prof. Dr. C. Bosch / Senckenberg-Museum Frankfurt/Main (FIS); (1 Ex.): Ganglb. 95, Bucsecs Tr. / Coll. Prof. Dr. C. Bosch / Senckenberg-Museum Frankfurt/Main (FIS); (1 Ex.): Ganglb. 95, 
Bucsecs Tr. (LMKK); (1 Ex.): Bucsecs Tr. / montana Kraatz (LMKK); (6 Ex.): Bucsecs, Deubel / alpin [Nicht als Paratypen etikettiert!] (coll. DeubeL: UTFS).

\section{Beschreibung}

Maße des Holotypus [in mm]: Körperlänge 3,44; Kopfbreite 0,51; Antennenlänge 1,10; Augenlänge 0,12; Schläfenlänge 0,24; Pronotumlänge 0,53; Pronotumbreite 0,66; Nahtlänge 0,30; Elytrenbreite 0,72; Abdomenbreite 0,72; Glied I der Hintertarsen 0,18; Glieder II bis IV der Hintertarsen zusammen 0,21; Aedoeaguslänge 0,54.

Farbe im ausgereiften Zustand pechbraun [immature \pm rotbraune Tiere sind häufig]. Kopf und Abdomen meist dunkler als der übrige Körper. Maxillarpalpen angedunkelt. Antennen und Beine düster rotbraun. Abdomenspitze nur undeutlich aufgehellt. Oberseite dicht chagriniert, nur matt glänzend. Punktur fein, im Chagrin schlecht sichtbar, auf dem Pronotum etwas deutlicher als auf Kopf und Elytren. Behaarung sehr fein und anliegend, auf dem Pronotum vom Typ III (im vorderen Fünftel der Mittellinie nach vorn, sonst nach hinten gerichtet), auf den Elytren fast gerade nach hinten gerichtet.

Körperlänge: 2,94-3,64 mm (MW 3,28; $\mathrm{n}=15$ ).

Kopf groß, aber viel schmaler als das Pronotum $(0,77)$; nach hinten gerundet erweitert, über den Schläfen am breitesten. Augen flach, aber etwas aus der Kopfrundung vorgewölbt, im Profil etwa die Hälfte der Schläfenlänge messend. Stirnnaht gerade. Schläfenrandung vollständig.

Antennen relativ schlank, zurückgelegt etwa die Mitte der Elytren erreichend. Maße der Antennen-

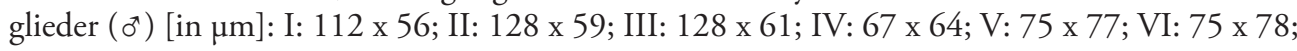
VII: 80 x 80; VIII: 80 x 82; IX: 80 x 88; X: 93 x 96; XI: 165 x 96.

Maxillarpalpen ziemlich gestreckt; Maße des Glieds III [im $\mu \mathrm{m}]: 152$ x 48.

Pronotum deutlich quer $(1,26)$, breiter als der Kopf $(1,29)$, etwas schmaler als die Elytren $(0,92)$; etwa in der Mitte am breitesten, nach vorn kaum stärker als nach hinten verengt. Vorder- und Hinterwinkel breit abgerundet. Vorderrand konvex, Hinterrand annähernd gerade. Fläche stärker gewölbt, mit einer \pm deutlichen Abflachung oder Andeutung einer Vertiefung in der Mitte. Hypomeren bei Ansicht im Profil sichtbar.

Elytren flach gewölbt, über den abgeschrägten Schultern etwas schmaler als das Pronotum, nach hinten fast geradlinig erweitert. Naht etwas vertieft, viel kürzer als die größte Breite $(0,42)$. Hinterrand zur Naht stumpfwinklig eingezogen, neben den Hinterwinkeln etwa in gleicher Tiefe eingebuchtet.

Abdomen kaum erweitert, über dem Segment V meist etwas breiter als die Elytren $(1,00-1,10)$. Tergite III bis V mit tiefen Basaleindrücken, Tergit VI mit deutlich schwächerem Basaleindruck. Tergit VII deutlich länger als Tergit VI, ohne Fransensaum.

Glied I der Hintertarsen mäßig gestreckt, etwas kürzer als die Glieder II bis IV zusammen $(0,86)$.

$0^{\star}$ : Aedoeagus (Fig. 26-27). Große Subapikalplatten relativ klein, kleiner als bei den anderen Arten. Paarige ventrale Fortsätze (Fig. 28). Apikalfortsatz der Paramere (Fig. 29), Subbasalhaar II ungewöhnlich lang.

ㅇ: Körper durchschnittlich etwas größer und breiter. Spermatheka (Fig. 30-31).

\section{Differentialdiagnose}

Tectusa bucegiensis sp. $\mathbf{n}$. wird mit T. transsylvanica sp. n. aus dem Fagaras-Massiv und mit T. rosenauensis sp. $\mathbf{n}$. vom Schuler-Gipfel verglichen. Sie ist etwas schmaler als T. transsylvanica, ihr 
Pronotum ist etwas stärker gewölbt, ihre Schultern sind stärker abgeschrägt, ihre Elytren stärker erweitert. Tectusa rosenauensis hat einen mehr gerundeten Kopf als T. bucegiensis, ihre Augen sind etwas stärker aus der Kopfrundung vorgewölbt.

\section{Bionomie}

Es sind nur die Sammelumstände bekannt. Der Holotypus und 118 Paratypen wurden aus Rasen und alpinen Polsterpflanzen am Schneefeldrand gesiebt, 107 Paratypen unter vergleichbaren Bedingungen, aber ohne Zusammenhang mit Schnee (Fig. 12). Drei Paratypen wurden subalpin gesiebt. Die Höhenangaben reichen von 1.800-2.300 m.

\section{Verbreitung}

Die neue Art ist bisher nur aus dem Bucegi-Gebirge bekannt und wahrscheinlich in diesem Massiv endemisch (Karte 2, S. 295). Da das Bucegi nach Westen aber nicht durch einen tiefen Einschnitt isoliert ist, kann man nicht ausschließen, dass T. bucegiensis auch in unmittelbar benachbarten Massiven vorkommt.

Untersuchte Exemplare: $80 \diamond^{\star} \diamond^{\star}, 24$ 우 ㅇ, 250 Ex.

Derivatio nominis: Den Namen bucegiensis (Adjektiv) wähle ich für diese Art aus dem BucegiMassiv in den Süd-Karpaten.

\subsection{Tectusa rosenauensis sp. $\mathrm{n}$.}

Fig. 7, 32-37, Karte 2

Oxypoda montana; Ganglbauer 1895: 76; Bernhauer 1902: 190 [104] et auctorum nec KraAtz, 1856: 187, ex parte.

\section{Typenmaterial}

Holotypus ( $\overbrace{}^{\star}$ ): Rosenauer-Gbg., Deubel [= Rosenauer Gebirge, leg. Deubel] / montana Kr. / O. Leonhard / HOLOTYPUS Tectusa rosenauensis Zerche (DEI).

Paratypen (1 \%): Rosenauer G., Deubel 96. [handschriftlich] / Oxypoda montana Kr. / CNHM 1955 Eduard Knirsch Palaearctic Colln. (CNHM); (1 ơ): Rosenauer-Gbg., Deubel / Oxypoda montana Kr. / CNHM 1955 Eduard Knirsch Palaearctic Colln. (CNHM); (1 ơ): RosenauerGbg., Deubel / montana Kr. / ex coll. Luze / Ost-Karpathen, Rosenauer-Gebirge (NHMW); (1 Ex.): Rosenauer Gbg., Täubl [handschriftlich; gemeint ist sicherlich DeubeL] / montana Kr. / ex coll. Luze (NHMW); (1 $\left.\sigma^{\top}\right)$ : Rosenauer-Gbg., Deubel / Bernh. don. / montana / coll. Schuster (NHMW); (1 ): Rosenauer-Gbg., Deubel / Bernh. det. / montana / coll. Schuster (DEI); (1 ㅇ ): Rosenauer G., Deubel / Bernh. don. / montana / coll. Schuster (NHMW); (1 $\left.0^{\star}\right)$ : $0^{\star} /$ RosenauGeb., Siebenbürg. / Deubel don. / Bernh. det. / montana / coll. Schuster (NHMW); (5 o o $\sigma^{\star}$, 1 o ): Rosenauer-Gbg. Deubel / Coll. Dr. Axel Krogh / Senckenberg-Museum Frankfurt/Main (FIS, DEI); $\left(2 \sigma^{*} o^{*}\right)$ : Rosenauer-Gbg. Deubel / Senckenberg-Museum Frankfurt/Main (FIS); $\left(2 \sigma^{\star} \sigma^{\star}\right)$ : Rosenauer-Gbg. Deubel / Deubel / Senckenberg-Museum Frankfurt/Main (FIS).

\section{Aus der Typenserie ausgeschlossen}

Die nachfolgenden fünf Tiere gehören zu T. rosenauensis, sind aber widersprüchlich etikettiert und schlecht erhalten (angeschimmelt). Die beiden an einer Nadel bzw. auf dem gleichen Etikett befindlichen Fundorte "Buczecz" und "Rosenauer Gebirge" schließen sich gegenseitig aus. Der letztere ist zutreffend. 
(1 ơ, 3 Ex.): Buczecz, Tr., Rosenauer Geb., Polonia (?) [handschriftlich] / Field. Mus. Nat. Hist.; 1966; A. Bierig Colln.; Acc. Z-13812 (CNHM); (1 Ex.): Buczecz, Tr., Rosenauer Geb., Polonia (?) [handschriftlich] / Rosenauer-Geb. Deubel [gedruckt] / BUCZECZ TR., SUBALPIN. [gedruckt] / Oxypoda montana [gedruckt; det. LoKay] / Field. Mus. Nat. Hist.; 1966; A. Bierig Colln.; Acc. Z-13812 (CNHM).

\section{Anmerkung zum locus typicus}

Hinter dem Namen „Rosenauer Gebirge“ [Rosenau = Râşnov] verbirgt sich wahrscheinlich das Schuler-Massiv mit dem Schuler-Gipfel, rumänisch Postăvaru bei Poiana Braşov, $1.799 \mathrm{~m}$, ca. $45^{\circ} 34^{\prime} \mathrm{N}, 25^{\circ} 34^{\prime} \mathrm{O}$. Es ist das von Rosenau aus nächstliegende Gebirge, das von Rosenau aus auch erreicht werden kann. Im Gipfelbereich hat Deubel (1910: 137) "Oxypoda montana" gesammelt. Deubel schreibt zwar "Schulergebirge", "Schulerhaus" oder "Schulerspitze"; es gibt jedoch bei dieser Art keine Käfer mit diesem Fundort, sondern immer nur die gedruckten Fundortzettel "Rosenauer Geb., Deubel". ${ }^{3}$ Die Zuordnung Rosenauer-Gebirge - Schuler ist nicht absolut eindeutig, weil es bei anderen Arten auch den gedruckten Fundort "Schuler-Gb., Deubel" gibt. Deshalb habe ich den ursprünglich konzipierten Namen "schulerensis" in rosenauensis verändert; eine falsche geographische Zuordnung soll auf jeden Fall vermieden werden. Ein eigener Versuch, die Art am 7.VI.2007 im Gipfelbereich des Schuler zu sammeln, blieb ohne Ergebnis.

Handschriftliche Etiketten, auch bei anderen Arten, sind stets von anderer Hand; Deubel hat oft mehrere Tiere an einer Nadel mit nur einem gedruckten Etikett präpariert.

\section{Beschreibung}

Maße des Holotypus [in mm]: Körperlänge 3,44; Kopfbreite 0,49; Antennenlänge 1,15; Augenlänge 0,11; Schläfenlänge 0,23; Pronotumlänge 0,52; Pronotumbreite 0,65 ; Nahtlänge 0,33; Elytrenbreite 0,70; Abdomenbreite 0,75; Glied I der Hintertarsen 0,17; Glieder II bis IV der Hintertarsen zusammen 0,16; Aedoeaguslänge 0,53.

Körperlänge: 2,88-3,60 mm (MW 3,26; $\mathrm{n}=13$ ).

Farbe düster rotbraun bis pechbraun. Kopf und Abdomen dunkler als der übrige Körper. Pronotum etwas heller, Elytren deutlich heller. Maxillarpalpen kaum angedunkelt. Antennen und Beine hell rotbraun. Vordere Tergite und Abdomenspitze nur undeutlich aufgehellt. Oberseite dicht und fein chagriniert, nur matt glänzend. Punktur fein, im Chagrin schwer sichtbar, auf dem Pronotum etwas deutlicher als auf dem Kopf, aber etwas schwächer als auf den Elytren. Behaarung sehr fein und anliegend, auf dem Pronotum vom Typ II (nach Benick \& LoHse 1974), auf den Elytren fast gerade nach hinten gerichtet.

Kopf groß, aber deutlich schmaler als das Pronotum (0,75); nach hinten gerundet erweitert, über den Schläfen am breitesten. Augen flach, kaum aus der Kopfrundung vorgewölbt, im Profil etwas weniger als die Hälfte der Schläfenlänge messend $(0,48)$. Stirnnaht etwas konvex. Schläfenrandung vollständig.

Antennen ziemlich schlank, zurückgelegt die Mitte der Elytren etwas übertreffend $\left(0^{\star}\right)$ oder diese erreichend ( $($ ) $)$. Maße der Antennenglieder ( $\sigma^{*}$ ) [in $\mu \mathrm{m}$ ]: I: 136 x 58; II: 128 x 56; III: 128 x 64; IV: 74 x 66; V: 67 x 80; VI: 72 x 82; VII: 80 x 85; VIII: 80 x 85; IX: 80 x 90; X: 81 x 96; XI: 168 x 99. Maxillarpalpen ziemlich gestreckt; Maße des Glieds III [in $\mu \mathrm{m}$ ]: 128 x 48.

\footnotetext{
${ }^{3}$ Auch im Rodnaer Gebirge hat er in seiner Publikation (Deubel 1910) die drei Berggipfel Kuhhorn, Koronjis und Saca genannt, nur bei wenigen Seltenheiten in der Artenliste auch den Gipfel angegeben. Aber alle seine Tiere tragen nur den gedruckten Zettel "Rodnaer Geb., Deubel”)
} 
Pronotum deutlich quer $(1,25)$, viel breiter als der Kopf $(1,33)$, kaum schmaler als die Elytren $(0,93)$; etwa in der Mitte am breitesten, nach vorn und hinten etwa gleich stark verengt. Vorderund Hinterwinkel kurz abgerundet. Vorderrand konvex, Hinterrand annähernd gerade. Fläche stärker gewölbt, mit einer \pm deutlichen schmalen, vorn etwas deutlicheren, Vertiefung in der Mitte. Hypomeren bei Ansicht im Profil sichtbar.

Elytren flach gewölbt, über den abgeschrägten Schultern etwas schmaler als das Pronotum, nach hinten in flachem Bogen erweitert. Naht etwas vertieft, viel kürzer als die größte Breite $(0,47)$. Hinterrand zur Naht stumpfwinklig eingezogen, neben den Hinterwinkeln etwa in gleicher Tiefe eingebuchtet.

Abdomen kaum erweitert, über dem Segment V etwas breiter als die Elytren $(1,07)$. Tergite III bis $\mathrm{V}$ mit tiefen Basaleindrücken, Tergit VI mit viel schwächerem Basaleindruck. Tergit VII deutlich länger als Tergit VI (ca. 1,35), ohne Fransensaum.

Glied I der Hintertarsen gestreckt, etwas kürzer als die Glieder II bis IV zusammen $(0,87)$.

$\sigma^{\star}$ : Aedoeagus (Fig. 32-33). Ventralfortsatz stark ventrad gebogen. Apex ziemlich spitz, in Ventralansicht als winziger Kreis innerhalb des breiten Aedoeagus-Körpers sichtbar. Paarige ventrale Fortsätze (Fig. 34). Apikalfortsatz der Paramere (Fig. 35).

: Spermatheka (Fig. 36-37).

\section{Differentialdiagnose}

Tectusa rosenauensis sp. $\mathbf{n}$. wird mit T. bucegiensis sp. $\mathbf{n}$. aus dem benachbarten Bucegi-Massiv und mit T. transsylvanica sp. n. aus dem Fagaras-Massiv verglichen. Sie unterscheidet sich von diesen Arten durch ihren kürzeren, stärker gerundeten Kopf und durch etwas aus der Kopfrundung vorgewölbte Augen.

\section{Bionomie}

Es sind nur die Sammelumstände bekannt: „Am Gipfel im Gesiebe aus Moos und Grasbüscheln, nicht selten (Deubel 1910 [Oxypoda montana]).“

\section{Verbreitung}

Die Art ist bisher nur aus dem Rosenauer Gebirge bekannt. Sehr wahrscheinlich verbirgt sich dahinter der isolierte Gipfel des Schuler [= Postăvaru]. Sie ist hier wahrscheinlich endemisch (Karte 2, S. 295).

Untersuchte Exemplare: $15 \sigma^{\star} \sigma^{\star}, 4$ 우 우, 5 Ex.

Derivatio nominis: Der Name rosenauensis (Adjektiv) nimmt auf den locus typicus Rosenauer Gebirge $[=$ Schuler $=$ Postăvaru bei Poiana Braşov $]$ in den Süd-Karpaten Bezug.

\subsection{Tectusa ceablauensis sp. n.}

Fig. 8, 38-41, Karte 2

Oxypoda montana; HoldHaus 1910: 116.

\section{Typenmaterial}

Holotypus ( $0^{\star}$ ): Ceahlau, Holdhaus / Ostkarpathen / Chicago NHMus M. Bernhauer Collection / montana Kr. Bukowina [Bernhauer script.] det. Bernhauer / HOLOTYPUS Tectusa ceahlauensis Zerche (CNHM). 
Anmerkung: Der Holotypus ist noch nicht ausgereift. Sein Aedoeagus ist im Bereich der Paramerenansätze beschädigt. Im Bereich der großen apikalen Platten ist das Präparat nicht durchsichtig, so dass hier nicht alle Innenstrukturen vollständig erkennbar sind.

\section{Beschreibung}

Maße des Holotypus [in mm]: Körperlänge 3,12; Kopfbreite 0,54; Antennenlänge 1,20; Augenlänge 0,10 ; Schläfenlänge 0,21 ; Pronotumlänge 0,59 ; Pronotumbreite 0,73 ; Nahtlänge 0,35; Elytrenbreite 0,80; Abdomenbreite 0,82; Glied I der Hintertarsen 0,22; Glieder II bis IV der Hintertarsen zusammen 0,20; Aedoeaguslänge 0,66.

Körper fast einheitlich hell rotbraun, nur die Tergite IV und V in der Mitte der Basalfurchen und das Tergit VI zu etwa zwei Dritteln gebräunt [Ausgereifte Tiere sind sicherlich deutlich dunkler.]. Oberseite sehr fein und undeutlich chagriniert, schwach glänzend, die Elytren nicht stärker glänzend. Punktur fein, zerstreut, auf Kopf und Elytren etwas stärker und deutlicher, auf dem Abdomen äußerst fein. Behaarung sehr fein und anliegend; auf dem Pronotum vom Typ III (nach Benick \& LoHse 1974); auf den Elytren annähernd gerade nach hinten gerichtet.

Kopf groß, aber deutlich schmaler als das Pronotum (0,74); nach hinten erweitert, deutlich über den gerundeten Schläfen am breitesten. Augen etwas aus der Kopfrundung vorgewölbt, klein, kaum halb so lang wie die Schläfen $(0,48)$. Stirnnaht gerade, fein, aber gut erkennbar. Schläfenrandung vollständig.

Antennen ziemlich schlank, zurückgelegt etwa das letzte Viertel der Elytren erreichend. Maße der Antennenglieder [in $\mu \mathrm{m}$ ]: I: 128 x 64; II: 128 x 56; III: 128 x 64; IV: 88 x 78; V: 80 x 77; VI: 82 x 85; VII: 83 x 90; VIII: 80 x 96; IX: 88 x 96; X: 88 x 99; XI: 195 x 96 .

Maxillarpalpen schlank, Glied III deutlich länger als der Pedicellus der Antennen (1,125); Proportionen des Glieds III [in $\mu \mathrm{m}$ ]: 144 x $56(2,57)$.

Pronotum deutlich quer $(1,24)$, viel breiter als der Kopf $(1,35)$, etwas schmaler als die Elytren $(0,91)$; etwa in der Mitte am breitesten; nach vorn kaum stärker als nach hinten verengt. Seitenränder fast gleichmäßig stark gebogen, nach hinten nur kaum merklich ausgeschweift. Vorderrand gerade. Hinterrand etwas konvex, vor dem Scutellum aber gerade. Hinterwinkel völlig geschwunden, kurz verrundet. Fläche stark gewölbt, nur im vorderen Fünftel mit der Andeutung einer Mittelfurche. Hypomeren wie bei allen Tectusa-Arten bei Ansicht im Profil sichtbar.

Elytren flach gewölbt, über den abgeschrägten Schultern etwas schmaler als das Pronotum, nach hinten fast geradlinig erweitert. Naht kürzer als die halbe Elytrenbreite $(0,44)$. Hinterrand zur Naht stumpfwinklig eingezogen, neben den Hinterwinkeln etwa in gleicher Tiefe eingebuchtet.

Abdomen bis zum Segment VI annähernd parallel. Tergite III bis V mit mäßig tiefem, Tergit VI mit flachem Basaleindruck. Tergit VII deutlich länger als Tergit VI $(1,37)$, ohne Fransensaum.

Glied I der Hintertarsen etwas länger als die Glieder II bis IV zusammen (1,05).

$0^{\star}$ : Antennenglied XI im Apikaldrittel nur schwach abgeschnürt. Aedoeagus (Fig. 38-39). Paarige ventrale Fortsätze sehr lang, ohne Ausschweifung (Fig. 40). Apikalfortsatz der Paramere (Fig. 41).

ㅇ: unbekannt.

\section{Differentialdiagnose}

Tectusa ceablauensis sp. $\mathbf{n}$. wird mit der räumlich nächsten Art T. rodnaensis sp. n. aus dem Rodnaer Gebirge verglichen. Tectusa ceablauensis hat schlankere Antennen und ein längeres Hintertarsenglied I, das länger als die Hintertarsenglieder II bis IV zusammen ist $(1,05)$. Ihr Pronotum ist etwa in der Mitte am breitesten. 


\section{Bionomie}

Es sind nur die Sammelumstände bekannt: „Oberhalb der Waldgrenze aus Grasbüscheln gesiebt (Holdhaus 1910 [Oxypoda montana]).“

\section{Verbreitung}

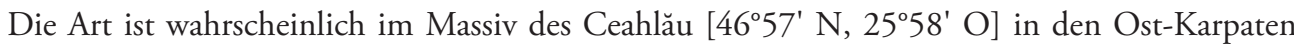
endemisch (Karte 2, S. 295).

Untersuchte Exemplare: nur Holotypus $\left(0^{*}\right)$.

Derivatio nominis: Der Name ceablauensis (Adjektiv) nimmt auf den locus typicus Munții Ceahlău in den Ost-Karpaten Bezug.

\subsection{Tectusa rodnaensis sp. $\mathrm{n}$.}

Fig. 9, 43-48, Karte 2

Oxypoda montana; Ganglbauer 1895: 76; 1896: 165; Bernhauer 1902: 190 [104] et auctorum nec KRAATZ, 1856: 187, ex parte.

\section{Typenmaterial}

Holotypus ( $\left.o^{\star}\right)$ : RO: Bistrița-Năsaud, Munții Rodnei, Gargalău-Massiv SO Borşa, 1.830 m,

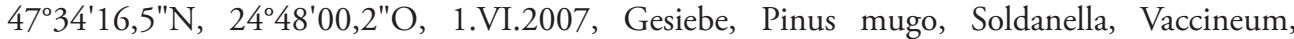
Juniperus, leg. Zerche / HOLOTYPUS Tectusa rodnaensis Zerche (DEI).

Paratypen (2 + o )): RO: Bistrița-Năsaud, Munții Rodnei, Gargalău-Massiv SO Borşa, 1.840 m, $47^{\circ} 34^{\prime} 22,6^{\prime \prime N}, 24^{\circ} 48^{\prime} 00,6^{\prime \prime}$, 1.VI.2007, Gesiebe, Pinus mugo, Soldanella, Rhododendron, Juniperus, leg. Zerche (DEI); (1 $\sigma^{*}, 1$ ㅇ [ursprünglich an einer Nadel]): Deubel, Rodnaer-Gb. [gedruckt] / alpin [gedruckt] / montana Kr. / Coll. Kraatz (DEI); (1 + ): Rodnaergb., Transsylv. / coll. Künnemann (DEI); (1 ơ, 1 क ): Ganglb. 96, Rodnaergb. (DEI); (2 ơ o , 3 Ex.): Ganglb. 96 / Rodnaergb. / coll. Koltze DEI (DEI); (2 $0^{\star} \sigma^{*}, 6$ 우 우): Rodnaergb., Transylv. / G. Fagel det. montana Kr. / [grüner Kreis] (IRSNB); (1 Ex.): Deubel, Rodnaer Gb. / alpin / G. Fagel det. montana Kr. / [grüner Kreis] (IRSNB); (1 , 2 Ex.): Deubel, Rodnaer Gb. / G. Fagel det. montana Kr. / [grüner Kreis] (DEI, IRSNB); (1 ơ 3 Ex.): Rodnaer Gb., Deubel / G. Fagel det. montana Kr. / [grüner Kreis] (IRSNB); (2 o $\sigma^{\star}, 2$ 우 ㅇ): Ganglb, 96, Rodnaergb. / G. Fagel det. montana Kr. / [grüner Kreis] (DEI, IRSNB); (3 o o ๑ $^{\star} 1$ , 4 Ex.): Rodnaer Gebirge, Deub. / Coll. Piesbergen (SMNS); (1 Ex.): Ganglb. 96, Rodnaergb / ex coll. Paganetti / Oxypoda montana Kr. det. E. Ulbrich 1990 / Sammlung Dr. Ulbrich (SMNS); (3 o o o $^{*} 1$ Ex.): Deubel, Rodnaer-Gb, / 71825 / Oxypoda montana Kr. (MNHUB); (1 , 3 Ex.): Deubel Tr., Rodnaer G. / Coll. Dr. Ihssen (MNHUB); (2 $o^{\star} o^{\star}, 1$ Ex.): Transsylvania, V. M. Duchon. / Rodnauer Gebirge / Oxypoda montana (MNHUB); (3 ㅇ ㅇ, 2 Ex.): Rodnaer-Geb. Tr. (MNHUB); (1 ơ, 2 ㅇ ㅇ, 1 Ex.): alpin / Deubel, Rodnaer-Gb. / Oxypoda montana Kr. (MNHUB); (2 우): Deubel. Tr., Rodna-Gb./ Oxyp. montana Kr. P. H. (MNHUB); (2 Ex.): Ünökö, Ost-Karpat., Götzelmann / Oxypoda montana (MNHUB); (1 ơ 1 Ex.): Rodnaer Geb. / Oxypoda montana, Deubel, Rodnaer Geb. (MNHUB); (1 ㅇ): Deubel, Rodnaer-Gb. / Lokay det. / 60 / Oxypoda montana (MNHUB); $\left(1 \mathrm{o}^{\top}\right)$ : Siebenbürgen, Rodnaer Geb. / Oxypoda montana / Coll. Hubenthal (MNHUB); (1 ơ, 1 ㅇ, 3 Ex.): Ganglb, 96, Rodnaergb / 67090 / Oxypoda montana (MNHUB); (1 ㅇ): L. carpathica, Rodnaer Geb. / Oxypoda sp. det. R. Pace 1979 (MNHUB); Deubel, Rodnaer-Gb. / ex coll. S. Stobiecki Inst. Zool. P. A. Kraków, 32/57 bzw. 45/57 (PANK); (2 o o , 1 ㅇ, 1 Ex.): Ganglb, 95, Rodnaergb / Prof. Schneider Vermächt. 1903 (MTD); (2 우 , 1 Ex.): Rodnaergebg., 


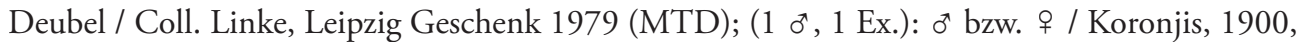
VII / Siebenbürgen / montana Kr. / ex coll. Scheerpeltz (NHMW); (1 $\left.\sigma^{*}\right)$ : Deubel, Rodnaer-Gb. / montana / coll. R. Dvořák (SNMB: Nicht als Paratypus etikettiert!); (1 $\left.0^{\star}\right)$ : Deubel, Rodnaer-Gb. / 46 / coll. V. Depta / Oxypoda montana (SNMB: Nicht als Paratypus etikettiert!); (2 Ex.): Tr. Óradna, Ünökö, Bokor (SNMB: Nicht als Paratypus etikettiert!); (1 Ex.): Deubel, Rodnaer-Gb. / montana Kr. / coll. Schuster (NHMW); (3 ơ o , 1 Ex.): Rodnaer-Geb., Tr. Schuster / montana Kr. / coll. Schuster (NHMW); (2 $\sigma^{\star} \sigma^{\star}, 6$ Ex.): Rodnaergeb., Deubel / montana Kr. [Bernhauer script.] / Chicago NHMus M. Bernhauer Collection (CNHM); (3 $\sigma^{\star} \sigma^{\star}, 3$ Ex.): Deubel, RodnaerGb. / Kuhhorn / Chicago NHMus M. Bernhauer Collection (CNHM); $\left(20^{\star} o^{\star}\right)$ : Kuhhorn / Chicago NHMus M. Bernhauer Collection (CNHM); (1 ơ, 1 Ex.): Siebenbg. / montana Kr., Rodnaergebg., ded. Schuster / Chicago NHMus M. Bernhauer Collection (CNHM); (1 $\left.0^{\star}\right)$ : Deubel, Rodnaer-Gb. / Oxypoda montana Kr. / CNHM 1955 Eduard Knirsch Palaearctic Colln. (CNHM); (1 ๙, 2 Ex.): Tr. Òradna, Ünökö, Bokor / montana K. det. Bernh. / Chicago NHMus M. Bernhauer Collection (CNHM); $\left(2 \sigma^{\star} o^{\star}\right)$ : Korongyos, Ost-Karpat., Götzelmann, montana Kr., det. Bernhau, don. Mader / Chicago NHMus M. Bernhauer Collection (CNHM); (1 q , 2 Ex.): Ünökö, Ost-Karpat., Götzelmann / Chicago NHMus M. Bernhauer Collection (CNHM); (3 ơ ơ, 1 + ): Ganglb. 96, Rodnaergb / montana Kr. / vid. Bernh. / ex coll. Skalitzky (1 Ex. mit dem zusätzlichen Etikett: Ost-Karpathen, Rodnaer-Gebirge) (NHMW); (5 Ex.): Ganglb. 96, Rodnaergb / montana Kr. / Bernh. determ. / ex coll. Scheerpeltz (NHMW); (1 ơ, 1 \%): wie vorige, aber: ex coll. Birnbacher (NHMW); (1 $\sigma^{*}, 1$ , 1 Ex.): wie vorige, aber: ex coll. Luze (NHMW); (2 ㅇ ๆ): Rodnaergb., Transsylv. / montana Kr. / ex coll. Scheerpeltz (NHMW); (1 o, 2 Ex.): Deubel, Rodnaer-Gb. / montana Kr. / ex coll. Scheerpeltz (NHMW); (2 Ex.): wie vorige, aber: vid. Bernh. / ex coll. Skalitzky (NHMW); (1 Ex.): Rodnaergebg., Deubel / montana Kr. / ex coll. Klima (NHMW); (1 o, 1 ㅇ ): Rodnaergb, Transsylv. / Coll. Achard Mus. Pragense (NMP); (21 Ex.): Deubel Tr., Rodnaer G. / Oxypoda montana (DEI, NMP); (5 o o $\sigma^{\star}, 148$ Ex.): Koroniez Tr., alpin. / Oxypoda montana (DEI, NMP); (76 Ex.): Koronec, Transsylv / Oxypoda montana (NMP); Deubel, Rodnaer-Gb. / Oxypoda montana Kr. / Coll. Prof. Dr. C. Bosch / SenckenbergMuseum Frankfurt/Main (FIS); (1 Ex.): Rodnageb. 1900.VII / 1110 / Hoffmann. / Coll. Prof. Dr. C. Bosch / Senckenberg-Museum Frankfurt/Main (FIS); (1 i ): Ost-Karpathen, Pietros [gedruckt; Gipfel im Rodnaer Gebirge] / Oxypoda montana det. E. Ulbrich 1985 / Sammlung Dr. Ulbrich (SMNS); (2 Ex.): Deubel, Rodnaer-Gb. / Kuhhorn (coll. Deubel: UTFS); (6 Ex.): Kuhhorn (coll. Deubel: UTFS); (3 $0^{\star} o^{\star}, 1$ Ex): Rodnaergeb. Transsylv. / Oxypoda montana / Ferdinandeum Innsbruck Slg. Reiss (TLFI).

\section{Beschreibung}

Maße des Holotypus [in mm]: Körperlänge 3,76; Kopfbreite 0,55; Antennenlänge 1,30; Augenlänge 0,14; Schläfenlänge 0,24; Pronotumlänge 0,58; Pronotumbreite 0,75; Nahtlänge 0,36; Elytrenbreite 0,81; Abdomenbreite 0,80; Glied I der Hintertarsen 0,19; Glieder II bis IV der Hintertarsen zusammen 0,20; Aedoeaguslänge 0,60.

Farbe in ausgereiftem Zustand dunkel rotbraun. Kopf kaum dunkler. Abdomen \pm geschwärzt, sein Apex nur undeutlich aufgehellt. Mundteile, Antennen und Beine gelbrot. Oberseite sehr dicht und deutlich chagriniert, nur matt glänzend. Punktur fein, im Chagrin schwer sichtbar, auf den Elytren etwas kräftiger und deutlicher erkennbar. Behaarung sehr fein und anliegend, auf dem Pronotum vom Typ II (nach Bеnicк \& Lohse 1974), auf den Elytren fast gerade nach hinten gerichtet.

Körperlänge: 3,20-4,32 mm (MW: 3,60; $\mathrm{n}=15$ ).

Kopf groß, aber merklich schmaler als das Pronotum (0,73-0,80); nach hinten gerundet erweitert, 
über den Schläfen am breitesten. Augen flach, aber etwas aus der Kopfrundung vorgewölbt, ihre Länge etwas variabel (Augen-Schläfen-Index 0,38-0,58). Stirnnaht flach konvex. Schläfenrandung vollständig.

Antennen ziemlich schlank, zurückgelegt die Mitte der Elytren etwas übertreffend. Maße der Antennenglieder ( $\sigma^{*}$ ) [in $\mu \mathrm{m}$ ]: I: 112 x 64; II: 128 x 62; III: 144 x 64; IV: 80 x 67; V: 82 x 80; VI: 83 x 83; VII: 83 x 83; VIII: 80 x 93; IX: 96 x 104; X: 96 x 104; XI: 168 x 104.

Maxillarpalpen gestreckt, Glied III viel länger als der Pedicellus der Antennen (1,31); Maße des Glieds III [in $\mu \mathrm{m}]: 168 \times 51$.

Pronotum deutlich quer (1,27-1,29), viel breiter als der Kopf (1,25-1,36), kaum schmaler als die Elytren (0,93); etwas hinter der Mitte am breitesten, nach vorn kaum stärker als nach hinten verengt. Seiten fast gleichmäßig gerundet. Vorder- und Hinterwinkel breit abgerundet. Vorderrand flach konvex, Hinterrand annähernd gerade. Fläche stärker gewölbt, mit einer Abflachung oder Andeutung einer Vertiefung in der Mitte. Hypomeren bei Ansicht im Profil sichtbar.

Elytren flach gewölbt, über den etwas abgeschrägten Schultern fast so breit wie das Pronotum, nach hinten in flachem Bogen erweitert. Naht schwach vertieft, viel kürzer als die größte Breite $(0,49)$. Hinterrand zur Naht stumpfwinklig eingezogen, neben den Hinterwinkeln etwa in gleicher Tiefe eingebuchtet.

Abdomen kaum erweitert, über dem Segment V etwa so breit wie die Elytren. Tergite III bis V mit tiefen Basaleindrücken, Tergit VI mit viel schwächerem Basaleindruck. Tergit VII wenig länger als Tergit VI, ohne Fransensaum.

Glied I der Hintertarsen gestreckt, etwa so lang wie die Glieder II bis IV zusammen $(0,95-1,05)$. $o^{\star}$ : Aedoeagus (Fig. 43-44). Ventralfortsatz im Profil ziemlich kräftig. Paarige ventrale Fortsätze (Fig. 45). Apikalfortsatz der Paramere (Fig. 46), Subbasalhaar I ungewöhnlich kurz.

ㅇ: Spermatheka (Fig. 47-48).

\section{Differentialdiagnose}

Tectusa rodnaensis sp. $\mathbf{n}$. ist von der nördlich in den ukrainischen Karpaten verbreiteten T. nigromontis sp. n. sicher nur durch die Genitalien zu trennen (Fig. 43-48, 49-54).

\section{Bionomie}

Die Art ist von der subalpinen bis in die alpine Höhenstufe verbreitet. Deubel (1910 [Oxypoda montana]) gibt an: „Von den Gipfeln des Kuhhorns und der Saca aus Grasbüscheln gesiebt“. Das Kuhhorn [= Ineu] ist mit 2.279 m der zweithöchste Gipfel im Gebirge. Der Holotypus und zwei Paratypen wurden im Bereich der Latschenkiefern (Pinus mugo) bei nur 1830-1840 m gesammelt.

\section{Verbreitung}

Die Art ist wahrscheinlich im Rodnaer Gebirge endemisch. Deubel (1910) sammelte sie auf den Gipfeln Kuhhorn [= Ineu = Ünökö] und Saca, alle seine Tiere sind aber nur mit "Rodnaer Gebirge" etikettiert (Karte 2, S. 295).

Untersuchte Exemplare: $54 \sigma^{\star} \sigma^{\star}, 36$ 우 우, 224 Ex.

Derivatio nominis: Der Name rodnaensis (Adjektiv) nimmt Bezug auf den locus typicus Rodnaer Gebirge in den Nordost-Karpaten. 


\subsection{Tectusa nigromontis sp. $\mathrm{n}$.}

Fig. 10, 49-54, Karte 2

Oxypoda montana; Reitter 1878: 38; Ganglbauer 1895: 76; Bernhauer 1902: 190 [104] et auctorum nec KraAtz, 1856: 187, ex parte.

\section{Typenmaterial}

Holotypus ( $\left.0^{\star}\right)$ : Czerna Hor., Reitter [handschriftlich] / Oxypoda montana, Czerna Hora [handschriftlich] / coll. Weise / [lila Quadrat] / HOLOTYPUS Tectusa nigromontis Zerche (DEI).

Paratypen (1 + ): Hoverla, Mazura / Oxypoda montana / Lokay det. (DEI); (1 ơ): Carpathes centr., Czarnohora, Obenberger / montana / ex coll. Rybiński, Inst. Zool. P. A. N. Kraków, 17/ 57 (PANK); (1 Ex.): Carpathes centr., Czarnohora, Obenberger / Alpin / montana / ex. coll. Scheerpeltz / Ost-Karpathen, Cerna hora, Hoverla (NHMW); (2 o o , 1 ㅇ , 4 Ex.): Galicya wsch., Czarna hora, M. Rybiński / Howerla 3/7 / montana / 18/850 / ex coll. Rybiński, Inst. Zool. P. A. N. Kraków, 34/57 (DEI, PANK); (9 Ex.): wie voriger, aber: 29.6. (PANK); (2 ㅇ , 2 Ex.): wie vorige, aber: Tomnatek,15.7. (DEI, PANK); (5 o $\sigma^{\star}, 8$ Ex.): wie vorige, aber: Dancerz, 15.7. (PANK); $\left(4 \sigma^{\top} \sigma^{\star}\right)$ : wie vorige, aber: Porzyzewska, 1.7. (DEI, PANK); (1 $\left.\sigma^{\star}\right)$ : wie vorige, aber: Nadgadzynor[?], 15/7 (PANK); (2 $\left.0^{\star} o^{\star}\right)$ : wie vorige, aber: Gadzyrsa [?], 29/6 (PANK); (3 o o , 4 Ex.): [schwarzes Quadrat] / 2537 / Oxypoda montana Kr. / Czarnohora, distr. Nadwórna, Howerla, leg. Stobiecki, 5/7.1923 (PANK); (1 ơ, 1 Ex.): wie vorige, aber: 2539 / ..., 7.1923 (PANK); (3 o o , 1 Ex.): wie vorige, aber: 1540-1542 / Halicia or., Czarnohora, Howerla, leg. Stobiecki, 12/6.1900 (PANK); (2 $\sigma^{\star} o^{\star}, 2$ Ex.): wie vorige, aber: 2585 / ... 8/7. (PANK); (1 $\left.\sigma^{\star}\right)$ : wie voriger, aber: 2589 / ... 9/7. (PANK); (1 $\left.\sigma^{\star}\right)$ : Ivan [gedruckt; = Pop Ivan] / G. Fagel det. montana Kr. / [grüner Kreis] (IRSNB); ( $\left.1 \sigma^{\top}\right)$ : Ivan [handschriftlich, ursprünglich sicherlich mit dem vorigen an einer Nadel], sonst wie voriger (IRSNB); $\left(2 \sigma^{\star} \sigma^{\top}\right)$ : Mármaros [gedruckt], P. Ivan [Pop Ivan, handschriftlich] / montana Kr., Hungaria, Dr. Causeur [handschriftlich] / c. Eppelsh. Steind. d. (NHMW, DEI); (1 ơ, 1 Ex.): Karpaty wsch, Dr. Lgocki / montana Kr. / ex coll. Scheerpeltz (NHMW); (4 Ex.): Karpaty wsch. Dr. Lgocki / Coll. Prof. Dr. C. Bosch / Senckenberg-Museum Frankfurt/Main (FIS); (1 Ex.): Carpathes centr., Czarnohora, Obenberger, Hoverla / Alpin / Oxypoda montana (SNMB: Nicht als Paratypus etikettiert!); (1 ơ , 8 Ex.): Podkar. Rus: Čorná hora, VII.23 / montana Roubal det. (SNMB: Nicht als Paratypus etikettiert!); (1 Ex.): Kúzy 32, Podk. Rus., Dr. Klička / Oxypoda montana det. Klička / coll. R. Dvořák (SNMB: Nicht als Paratypus etikettiert!); (2 Ex.): Carpathes centr., Czarnohora, Obenberger / Alpin / Czarnohora Polonia / Oxypoda montana / Field Mus. Nat. Hist. 1966 A. Bierig Colln., Acc. Z-13812 (CNHM); (2 Ex.): Hoverla-Pietrosz, 25.6.1923 / Carpath. or., J. MATCHA / Oxypoda montana Kr. (NMP); (17 o o , 4 우 ㅇ, 11 Ex.): Hoverla-Pietrosz, 25.6.1923 / Carpath. or., J. MATCHA (DEI, NMP); (1 ơ 1 Ex.): Carpath. Gutin, Tomnatek, 30.VIII.24, Dr. Rambousek (NMP); (1 Ex.): Carpathes centr., Czarnohora, Obenberger / Coll. Procházka / Bessopora montana (NMP); (1 $\left.\sigma^{\star}\right)$ : Carpathes centr., Czarnohora, Obenberger / Alpin / Oxypoda (Besso-pora) montana (NMP); (5 $0^{\star} \sigma^{*}, 3$ o 우): Porzyžewska, Cp. or., 11908 / Oxypoda montana (NMP); (3 o o , 5 우 ㅇ): Czarnohora, Cp. Or., 10908 / Oxypoda montana (NMP); (1 $\sigma^{\star}, 1$ ㅇ): Zakarpatska obl., Rahiv distr., Karpatsky biosferni zapovidnik [Reserve], Chornohora mts., Jan Růžička leg. / polonina Breskul $\rightarrow$ Hoverla mt., 6.VI.1999, 1.650-1.800 m, sifted old remnants of grasses, margins of snow fields / Oxypoda sp., St. Snäll det. 2002 (coll. RŮŽIČKA; DEI); (1 †): wie vorige, aber polonina Breskul, cascade -> gap under Petros mt., 1500-1900 m, 30.viii.1999, along path, excr. of Bos, pastures + coniferous forests (coll. RŮŽIČKA). 


\section{Beschreibung}

Maße des Holotypus [in mm]: Körperlänge 3,52; Kopfbreite 0,54; Antennenlänge 1,29; Augenlänge 0,12; Schläfenlänge 0,23; Pronotumlänge 0,58; Pronotumbreite 0,74; Nahtlänge 0,39; Elytrenbreite 0,80; Abdomenbreite 0,79; Glied I der Hintertarsen 0,21; Glieder II bis IV der Hintertarsen zusammen 0,18; Aedoeaguslänge 0,58.

Körperlänge: 3,04-4,24 (MW 3,69; n = 15).

Farbe in ausgereiftem Zustand dunkel rotbraun, meist jedoch nicht ausgereift und \pm heller. Kopf etwas dunkler. Abdomen \pm umfangreich geschwärzt, sein Apex nur undeutlich aufgehellt. Mundteile, Antennen und Beine gelbrot. Oberseite sehr dicht und deutlich chagriniert, nur matt glänzend. Punktur fein, aber gut erkennbar, auf den Elytren etwas stärker. Behaarung sehr fein und anliegend, auf dem Pronotum vom Typ II, auf den Elytren fast gerade nach hinten gerichtet.

Kopf groß, aber viel schmaler als das Pronotum (0,72); nach hinten gerundet erweitert, über den Schläfen am breitesten. Augen flach, kaum aus der Kopfrundung vorgewölbt, im Profil etwa die Hälfte der Schläfenlänge messend. Stirnnaht gerade. Schläfenrandung vollständig.

Antennen ziemlich schlank, zurückgelegt das letzte Drittel der Elytren erreichend $\left(\sigma^{\star}\right)$. Maße der Antennenglieder ( $\left.\sigma^{\top}\right)$ [in $\mu \mathrm{m}$ ]: I: 117 x 64; II: 128 x 58; III: 134 x 62; IV: 80 x 67; V: 78 x 77; VI: 88 x 80; VII: 93 x 85; VIII: 96 x 85; IX: 101 x 95; X: 118 x 109; XI: 176 x 106.

Maxillarpalpen gestreckt, Glied III viel länger als der Pedicellus der Antennen $(1,40)$; Maße des Glieds III [in $\mu \mathrm{m}]: 179 \times 55$.

Pronotum deutlich quer $(1,26)$, viel breiter als der Kopf $(1,39)$, kaum schmaler als die Elytren $(0,93)$; kurz hinter der Mitte am breitesten, nach vorn kaum stärker als nach hinten verengt. Seiten fast gleichmäßig gerundet. Vorder- und Hinterwinkel breit abgerundet. Vorderrand flach konvex, Hinterrand annähernd gerade. Fläche stärker gewölbt, meist mit einer Abflachung oder Andeutung einer Vertiefung in der Mitte, manchmal nur im hinteren Bereich. Hypomeren bei Ansicht im Profil sichtbar.

Elytren flach gewölbt, über den kaum abgeschrägten Schultern fast so breit wie das Pronotum, nach hinten fast geradlinig schwach erweitert. Naht schwach vertieft, viel kürzer als die größte Breite $(0,46)$. Hinterrand zur Naht stumpfwinklig eingezogen, neben den Hinterwinkeln etwa in gleicher Tiefe eingebuchtet.

Abdomen kaum erweitert, über dem Segment V etwa so breit wie die Elytren. Tergite III bis V mit tiefen Basaleindrücken, Tergit VI mit deutlich schwächerem Basaleindruck. Tergit VII deutlich länger als Tergit VI $(1,41)$, ohne Fransensaum.

Glied I der Hintertarsen gestreckt, etwas länger als die Glieder II bis IV zusammen $(1,17)$.

$\sigma^{*}$ : Aedoeagus (Fig. 49-50). Apex des Ventralfortsatzes breit, gerade abgestutzt. Paarige ventrale Fortsätze (Fig. 51). Apikalfortsatz der Paramere (Fig. 52).

: Spermatheka (Fig. 53-54).

\section{Differentialdiagnose}

Tectusa nigromontis sp. $\mathbf{n}$. hat längere Antennen als T. rodnaensis sp. $\mathbf{n}$. aus dem südlich benachbarten Rodnaer Gebirge. Sicher zu trennen sind beide Arten nur durch Genitalvergleich (Fig. 43-48, 49-54). 


\section{Bionomie}

ReITter (1878) hat in der Einleitung zum Reisebericht und unter Oxypoda montana die Sammelumstände mitgeteilt: "Im Juni 1877" ... "fuhren [wir] über Luhy zur Howerla- und Salvatorklause an der Howerla-Alpe (Czerna Hora) gelegen.” ... "An Schneerändern der Czerna Hora. Leder fing ein Stück unter einem Steine. Eine weitere Anzahl hatten wir dem Umstande zu danken, dass wir Rasenstücke, hart am thauenden Schnee, abstachen und in unser Standquartier auf Pferden schleppen ließen.”

Die letztgenannten aktuellen Aufsammlungen sind wahrscheinlich nicht typisch für das Vorkommen dieser Art.

\section{Verbreitung}

Die Art ist ein Endemit des Chornohora [= Czerna hora]-Massivs in den ukrainischen Karpaten. Ihr Areal reicht wahrscheinlich bis in die Munții Marmamureşului nach Rumänien, denn es gibt auch Nachweise vom Grenzberg zwischen der Ukraine und Rumänien, dem Pop Ivan (Karte 2, S. 295).

Untersuchte Exemplare: $59 \sigma^{\star} \sigma^{\star}, 18$ 우 우, 58 Ex.

Derivatio nominis: Der Name nigromontis (Substantiv im Genitiv; des schwarzen Berges: eine Kombination aus niger, Lat. schwarz, und mons, Lat. Berg) bezieht sich auf den locus typicus, das Chornohora-Massiv in den Nordost-Karpaten.

\section{Tabelle der Tectusa-Arten der Karpaten}

1 Antennen etwas länger. Körper durchschnittlich etwas größer. Körperlänge 3,04-4,24 (MW 3,69). Habitus (Fig. 10). Aedoeagus (Fig. 49-50) in Ventralansicht mit breitem Apex, der gerade abgestutzt ist. Apikalfortsatz der Paramere (Fig. 51). Paarige ventrale Fortsätze des Aedoeagus (Fig. 52). Spermatheka (Fig. 53-54). Ukraine, Chornohora, Pop Ivan (Karte 2). T. nigromontis sp. $\mathbf{n}$.

Antennen etwas kürzer. Körper durchschnittlich etwas kleiner. Körperlänge 2,88-4,20 (MW von 3,26-3,59). Aedoeagus (Fig. 21, 27, 33, 39, 44) in Ventralansicht mit schmalem rundlichen Apex. Rumänien. 2

2 Kopf stärker gerundet und etwas kürzer. Die kleinen Augen etwas aus der Kopfkontur vorgewölbt. Körperlänge 2,88-3,60 mm (MW 3,26). Habitus (Fig. 7). Aedoeagus (Fig. 32 33), Apikalteil stärker ventrad gekrümmt, Apex als rundlicher Punkt innerhalb des breiteren Aedoeagus vorstehend. Apikalfortsatz der Paramere (Fig. 34). Paarige ventrale Fortsätze des Aedoeagus (Fig. 35). Spermatheka (Fig. 36-37). Rumänien, Süd-Karpaten, Schuler-Gipfel = Postăvaru bei Poiana Brașov (Karte 2). T. rosenauensis $\mathbf{s p .} \mathbf{n}$.

Kopf schwächer gerundet und etwas länger. Die Augen nicht oder nur sehr schwach aus der Kopfkontur vorgewölbt. Apikalteil des Aedoeagus schwächer ventrad gekrümmt, Apex rundlich abgesetzt und stets den breiteren Aedoeagus etwas überragend, ein angedeuteter Stiel erkennbar (Fig. 21, 27, 39, 44). 3 
3 Körperlänge 3,12 mm. Habitus (Fig. 8). Aedoeagus (Fig. 38-39). Apikalfortsatz der Paramere (Fig. 40). Paarige ventrale Fortsätze des Aedoeagus gerade, lang zugespitzt und länger als bei den anderen Arten (Fig. 41). ㅇ unbekannt. Ost-Karpaten, Ceahlau-Massiv (Karte 2). T. ceablauensis sp. $\mathbf{n}$.

- $\quad$ Paarige ventrale Fortsätze des Aedoeagus kürzer, deutlich geschweift oder gerade, aber nicht lang zugespitzt.

4 Schultern stärker reduziert, die Seiten der Elytren deshalb mehr abgeschrägt. Durchschnittlich kleiner. Körperlänge 2,94-3,64 mm (MW 3,28). Habitus (Fig. 6). Aedoeagus (Fig. 26-27). Apikalfortsatz der Paramere (Fig. 28). Paarige ventrale Fortsätze des Aedoeagus (Fig. 29) kurz, vor dem apikalen Drittel ausgeschweift, subapikal nach außen erweitert und relativ kurz zugespitzt. Spermatheka (Fig. 30-31). Süd-Karpaten, Bucegi (Karte 2).

T. bucegiensis sp. $\mathbf{n}$.

Durchschnittlich größer. Schultern weniger reduziert, die Seiten der Elytren etwas weniger abgeschrägt. Paarige ventrale Fortsätze des Aedoeagus kurz, ziemlich breit und ohne Ausschweifung (Fig. 45) oder lang, in der Mitte ausgeschweift und lang zugespitzt (Fig. 22). 5

5 Körperlänge 3,05-4,20 mm (MW 3,51). Habitus (Fig. 5). Aedoeagus (Fig. 20-21). Apikalfortsatz der Paramere (Fig. 22), Subbasalhaar I des Fortsatzes fast halb so lang wie das Subbasalhaar II (47\%). Paarige ventrale Fortsätze des Aedoeagus (Fig. 23) lang, in der Mitte ausgeschweift und lang zugespitzt. Spermatheka (Fig. 24-25). Süd-Karpaten, Făgăraş (Karte 2). T. transsylvanica sp. $\mathbf{n}$.

- Körperlänge 3,20-4,32 (MW: 3,60 mm). Habitus (Fig. 9). Aedoeagus (Fig. 43-44). Apikalfortsatz der Paramere (Fig. 46), Subbasalhaar I des Fortsatzes ungewöhnlich kurz, nur etwa 27 \% der Länge des Subbasalhaars II messend. Paarige ventrale Fortsätze des Aedoeagus (Fig. 45) kurz, ziemlich breit und ohne Ausschweifung. Spermatheka (Fig. 47-48). NordostKarpaten, Rodnaer Gebirge (Karte 2). T. rodnaensis sp. $\mathbf{n}$.

\section{Literatur}

Assing, V. \& SchüLke, M. 2001: Supplemente zur mitteleuropäischen Staphylinidenfauna (Coleoptera, Staphylinidae). II. - Entomologische Blätter, Keltern 97: 121-176.

Assing, V. \& SchüLke, M. 2007: Supplemente zur mitteleuropäischen Staphylinidenfauna (Coleoptera, Staphylinidae). III. - Entomologische Blätter, Schwanfeld 102 (2006) (1-3): 1-78.

Benick, G. \& Lohse, G. A. 1974 (72-220): 14. Tribus Callicerini (Athetae). - In: Freude, H.; Harde, K. W. \& Lohse, G. A. (Hrsg.): Die Käfer Mitteleuropas. Bd. 5. Staphylinidae II (Hypocyphtinae und Aleocharinae). Pselaphidae. - Krefeld; Goecke \& Evers: 381 S.

Bernhauer, M. 1902: Die Staphyliniden der paläarktischen Fauna. I. Tribus: Aleocharini. (II. Theil). Verhandlungen der zoologisch-botanischen Gesellschaft Wien 52, Beiheft: 87-284 [Separatum: 1-198].

BoнÁč, J. 1993 (39-62): Staphylinidae. - In: Jelínek, J. (Hrsg.): Check-list of Czechoslovak Insects IV (Coleoptera). - Folia Heyrovskyana, Supplementum 1, Praha: 172 S.

Burakowski, B.; Mroczkowski, M. \& Stephańska, J. 1981: Katalog Fauny Polski. XXIII, 8. Staphylinidae 3. - Warszawa; Państwowe Wydawnictwo Naukowe: 330 S., 1 Karte.

Deubel, F. 1910 (126-200): C. Die Coleopterenfauna des Csukás, Schuler, Bucsecs, Königstein, Bulea-Sees, Negoi, Retyezát, Paring und des Rodnaer Gebirges. - In: Holdhaus, K. \&Deubel, F.: Untersuchungen über die Zoogeographie der Karpaten (unter besonderer Berücksichtigung der Coleopteren). Abhandlungen der zoologisch-botanischen Gesellschaft Wien 6 (1): IV + 202 S., 1 Karte.

Diepenbroek, M.; Grobe, H. \& Sieger, R. 2000: PanMap. http://www.pangaea.de/ Software/PanMap 
Fenyes, A. 1920: Coleoptera. Fam. Staphylinidae. Subfam. Aleocharinae. - In: Wytsman, P. (Hrsg.): Genera Insectorum. - La Haye: Fasc. 173A, 1918: 1-110; - Tervuren: Fasc. 173B, 1920: 111-414; Fasc. 173C, 1921: 415-453, 7 Taf.

Ganglbauer, L. 1895: Die Käfer von Mitteleuropa. Die Käfer der österreichisch-ungarischen Monarchie, Deutschlands, der Schweiz, sowie des französischen und italienischen Alpengebietes. 2. Bd. Familienreihe Staphylinoidea. 1. Teil: Staphylinidae, Pselaphidae. - Wien; Carl Gerold's Sohn: 880 S.

Ganglbauer, L. 1896: Sammelreisen nach Südungarn und Siebenbürgen. Coleopterologische Ergebnisse derselben. I. Theil. - Annalen des K. K. Naturhistorischen Hofmuseums, Wien 11 (2): 164-187.

Gemminger \& Harold, B. De 1868: Familia VI. Staphylinidae. - In: Catalogus Coleopterorum hucusque descriptorum synonymicus et systematicus. Tom II. - Monachii; E. H. Gummi: 502-680.

Holdhaus, K. 1910 (I-IV, 1-125, 201-202, 1 Karte): Einleitung. A. Allgemeine Untersuchungen über die Zoogeographie der Karpathen. B. Beiträge zur Kenntnis der Coleopterenfauna der Ostkarpathen. Nachträge. Bemerkungen zur Karte. - In: Holdhaus, K. \& Deubel, F.: Untersuchungen über die Zoogeographie der Karpaten (unter besonderer Berücksichtigung der Coleopteren). - Abhandlungen der zoologisch-botanischen Gesellschaft Wien 6 (1): IV + 202 S., 1 Karte.

Horion; A. 1967: Faunistik der Mitteleuropäischen Käfer. Bd. 11. Staphylinidae. 3. Teil: Habrocerinae bis Aleocharinae (Ohne Subtribus Athetae). - Überlingen - Bodensee: XXIV + 419 S.

KraAtz, G. 1856: Naturgeschichte der Insecten Deutschlands. Bd. 2. Staphylinii. - Berlin; Nicolai: VIII + 1080 S.: 1-376 [31.03.1856] , 377-768 [31.05.1857] , 769-1080 (30.11.1857), I-VIII (1858).

KraAtz, G. 1859: In: Neuere Literatur. - Genera des Coléoptères par M. Jaquelin du Val et M. J. MigneauX. Livrasion 45-61. - Berliner entomologische Zeitschrift 3: IV-IX.

Kraatz, G. 1869: Verzeichniß der Käfer Deutschlands (Herausgegeben von dem Entomologischen Verein in Berlin). - Berlin; Nicolai: 84 S.

Lohse, G. A. 1974 (230-291): 18. Tribus Oxypodini. - In: Freude, H.; Harde, K. W. \& Lohse, G. A. (Hrsg.): Die Käfer Mitteleuropas. Bd. 5. Staphylinidae II (Hypocyphtinae und Aleocharinae). Pselaphidae. - Krefeld; Goecke \& Evers: 381 S.

Lohse, G. A. 1989 (185-240): Ergänzungen und Berichtigungen zu Freude-Harde-Lohse „Die Käfer Mitteleuropas" Band 5 (1974). - In: Lohse, G. A. \& Lucht, W. H. (Hrsg.): Die Käfer Mitteleuropas. 1. Supplementband mit Katalogteil. - Krefeld; Goecke \& Evers: 346 S.

Neuhäuser-Happe, L. 1999 (291-345): Rote Liste der Kurzflügelkäfer Kärntens (Insecta: Coleoptera: Staphylinoidea: Staphylinidae). - In: Rottenburg, T.; Wieser, C.; Mildner, P. \& Holzinger, W. E. (Hrsg.): Rote Listen gefährdeter Tiere Kärntens. - Naturschutz in Kärnten, Klagenfurt 15: 718 S.

Petri, K. 1912: Siebenbürgens Käferfauna aufGrund ihrer Erforschung bis zum Jahre 1911 (Siebenbürgischer Verein für Naturwissenschaften Hermannstadt). - Berlin; R. Friedländer \& Sohn: X + 376 S.

Porta, A. 1926: Fauna Coleopterorum Italica. Vol. 2. - Staphylinoidea. Staphylinidae, Pselaphidae, Clavigeridae, Scydmaenidae, Silphidae, Liodidae, Clambidae, Leptinidae, Platypsyllidae, Corylophidae, Sphaeriidae, Trichopterygidae, Hydroscaphidae, Scaphidiidae, Histeridae. - Piacenza; Stabilimento Tipographico Piacentino: 405 S.

Redtenbacher, L. 1856: Fauna austriaca. Die Käfer. Nach der analytischen Methode bearbeitet. Ed. 2. - Wien; Carl Gerold's Sohn (1856-1858): - CXXXVI S. (1858) + 1-976 (1856 [24.09.]), 977-1017, 2 Taf. (1858).

Redtenbacher, L. 1872: Fauna austriaca. Die Käfer. Nach der analytischen Methode bearbeitet. Ed. 3. - Wien; Carl Gerold's Sohn (1872-1874): - Bd. 1.: CLIII S. (1874) + 564 S. (1872). - Bd. 2. 571 S., 2 Taf. (1874).

Reitter, E. 1878: Beitrag zur Coleopteren-Fauna der Carpathen. - Deutsche entomologische Zeitschrift, Berlin 22: 33-64.

\footnotetext{
${ }^{4}$ Datiert in: SCHAUм (1856: 3).

${ }^{5}$ Datiert in: KraAtz (1859: VII, nota 2).
} 
Roubal, J. 1930: Katalog Coleopter (Brouků) Slovenska a Podkarpatska na základě bionomickém a zoogeografickém a spolu systematický doplněk Ganglbauerových "Die Käfer von Mitteleuropa" a Reitterovy "Fauna germanica". I. - Praha: 527 S.

Schaum, H. 1856: [Prospectus über „Naturgeschichte der Insecten Deutschlands“; eingebunden in Allgemeine Deutsche Naturhistorische Zeitung, N. F., Dresden 3 (1857) der Bibliothek des DEI]. $3 \mathrm{~S}$.

Scheerpeltz, O. 1958: Neue Arten der Gattung Parocyusa Bernh., nebst einer Bestimmungstabelle der bis heute bekannt gewordenen Arten dieser Gattung (Coleoptera, Staphylinidae). (31. Beitrag zur Kenntnis der paläarktischen Staphylinidae). - Nachrichtenblatt Bayerischer Entomologen, München 7: 102-112.

Smetana, A. 2004 (353-494): Subfamily Aleocharinae Fleming, 1821. - In: Löbl, I. \& Smetana, A. (Hrsg.): Catalogue of Palaearctic Coleoptera, Vol. 2. - Stenstrup; Apollo Books: 942 S.

WinkLeR, A. 1925: Catalogus coleopterorum regionis palaearcticae. Pars 4. - Wien; Albert Winkler: 369-496.

Zerche, L. 1990: Monographie der paläarktischen Coryphiini (Coleoptera, Staphylinidae, Omaliinae). - Berlin; Akademie der Landwirtschaftswissenschaften: 413 S.

Zerche, L. 1993: Monographie der paläarktischen Coryphiini (Coleoptera, Staphylinidae, Omaliinae). - Supplementum 1. - Beiträge zur Entomologie, Berlin 43 (2): 319-374.

\section{Anschrift des Verfassers:}

Dr. Lothar Zerche

Deutsches Entomologisches Institut (DEI)

Leibniz-Zentrum für Agrarlandschaftsforschung (ZALF)

Eberswalder Str. 84

15374 Müncheberg, Germany

E-Mail: zerche@zalf.de

\section{Subject editor:}

Prof. Dr. B. Klausnitzer 

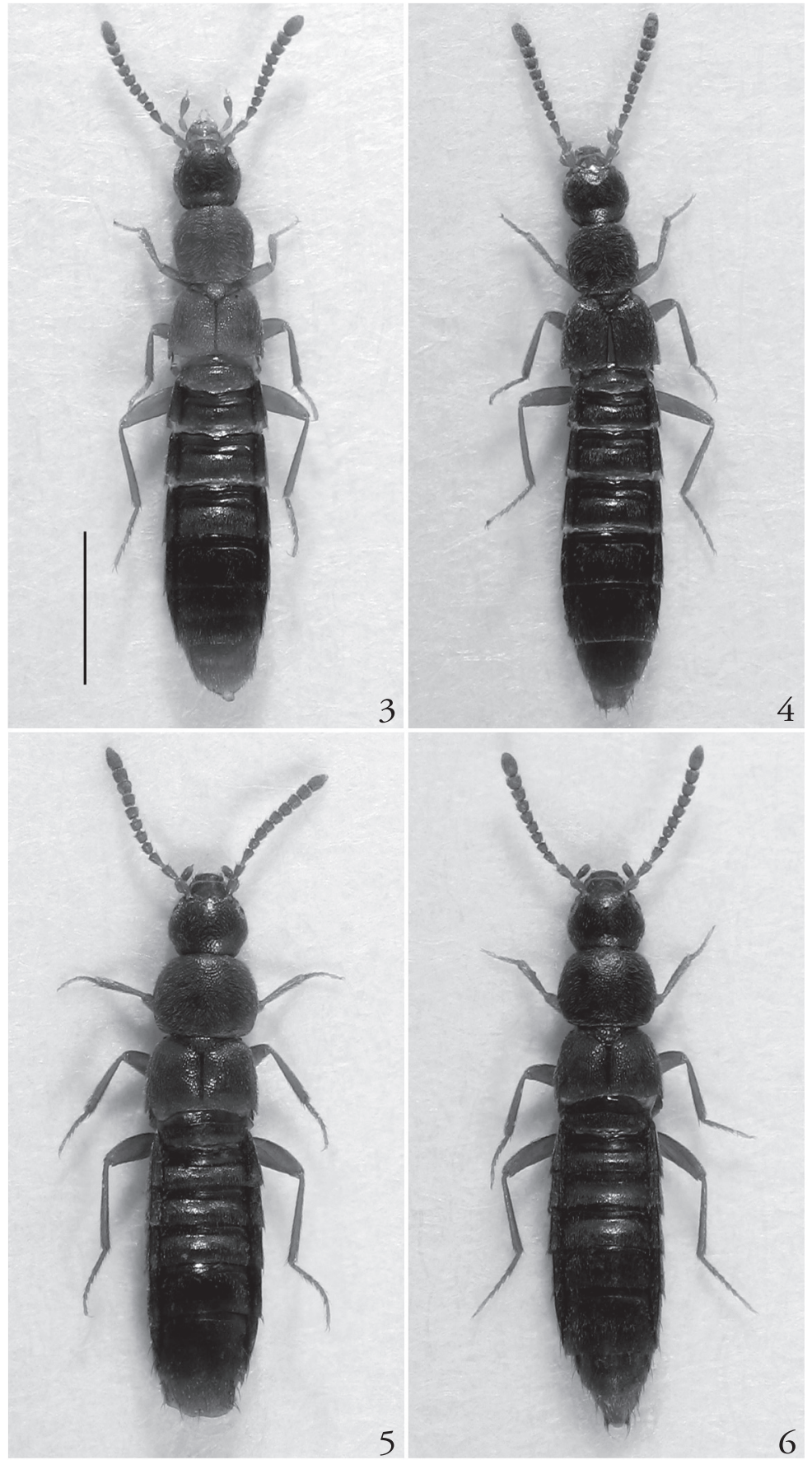

Fig. 3-6: Habitus von Tectusa-Arten [Maßstab 1 mm]. -3 T. montana (KraAtz) [ (Bernhauer) [o, Topotypus]. 5 T. transsylvanica sp. n. [Holotypus]. 6 T. bucegiensis sp. n. [Holotypus]. 


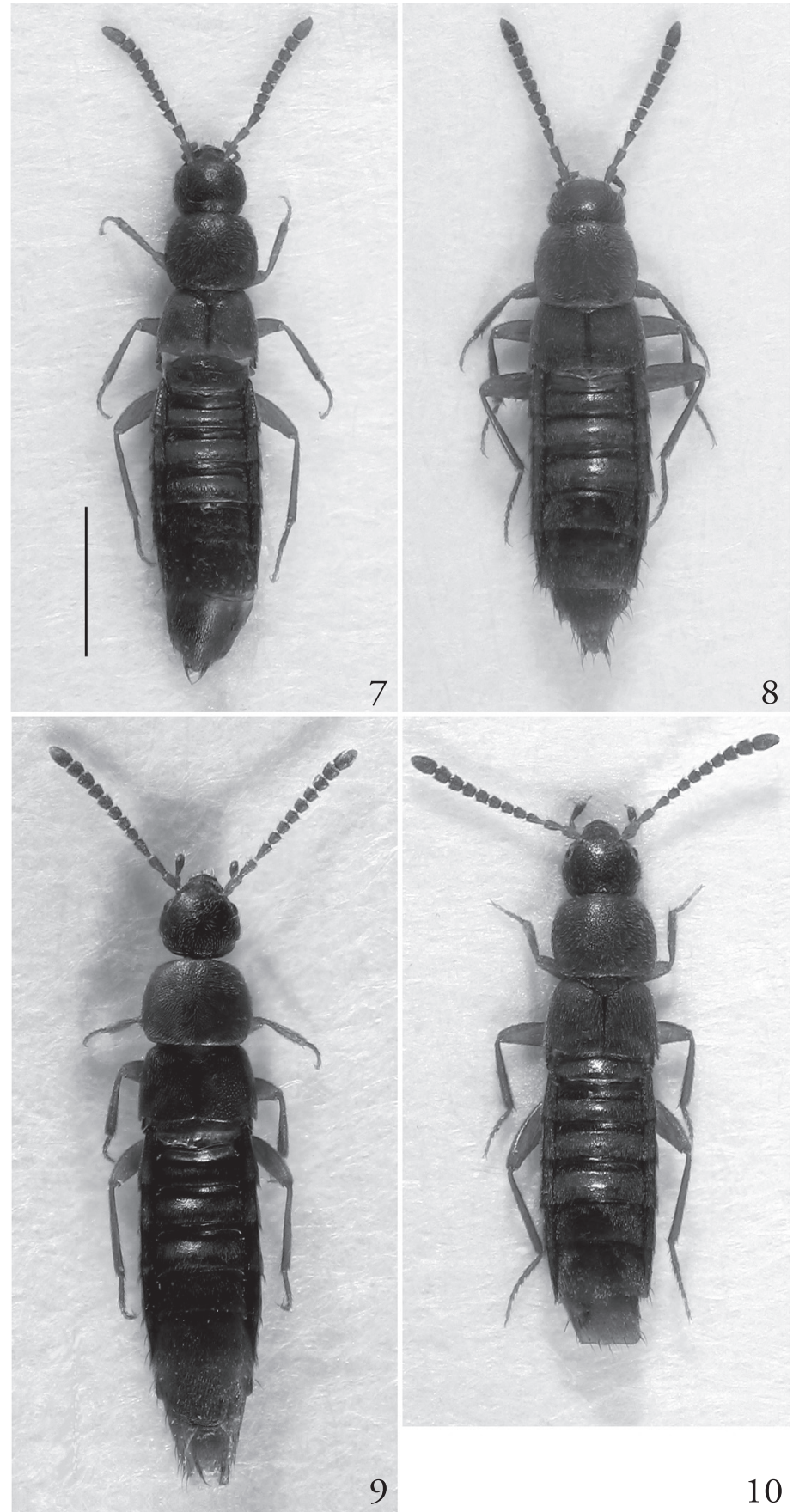

Fig. 7-10: Habitus von Tectusa-Arten [Maßstab 1 mm]. - 7 T. rosenauensis sp. n. [Holotypus]. 8 T. ceablauensis sp. n. [Holotypus]. 9 T. rodnaensis sp. n. [Holotypus]. 10 T. nigromontis sp. n. [Holotypus]. 

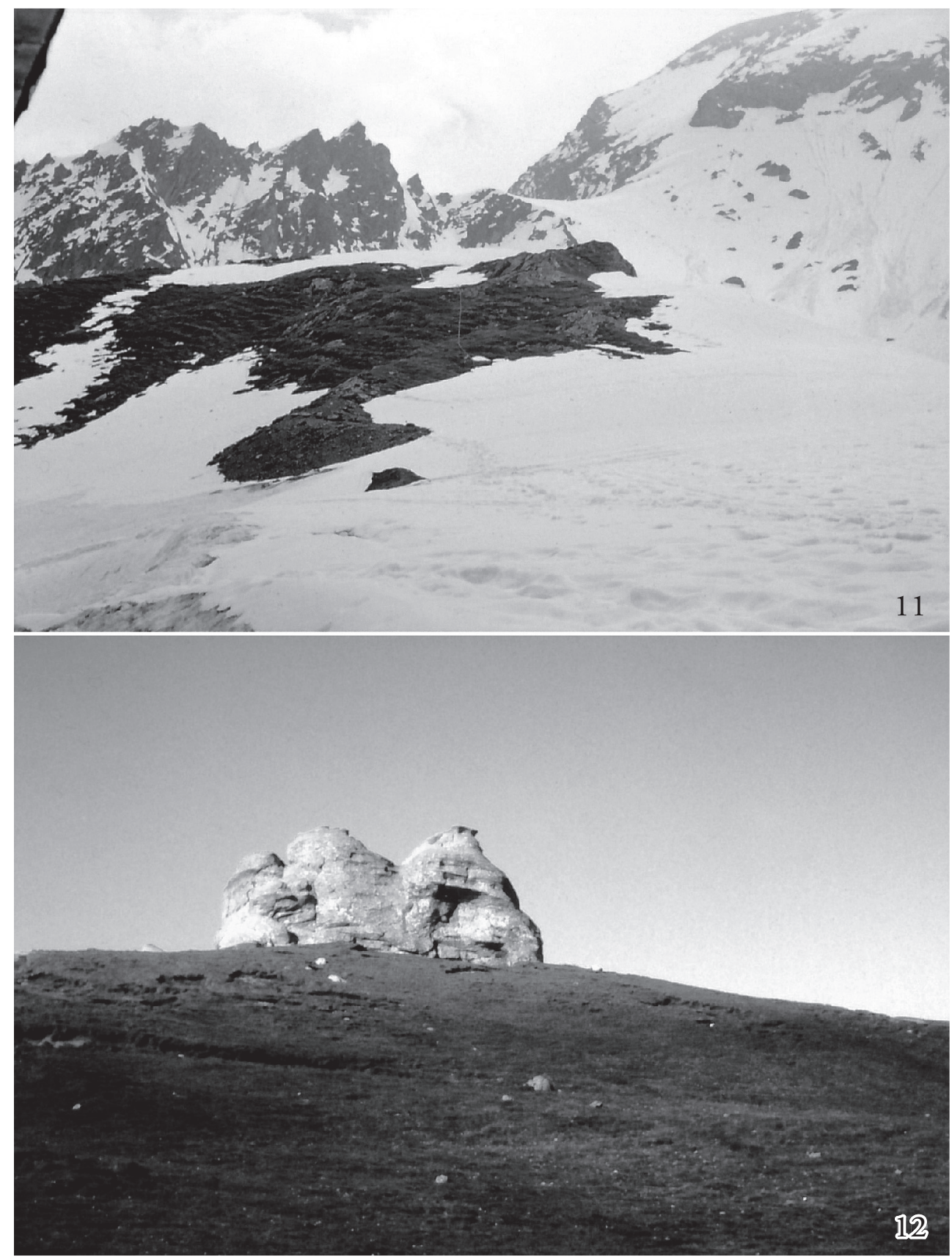

Fig. 11: Locus typicus von Tectusa transsylvanica oberhalb des Buleasees im Făgăraş-Massiv [rechts im Hintergrund].

Fig. 12: Alpine Rasen um Felsen - Sammellokalität von Tectusa bucegiensis im Bucegi-Massiv. 


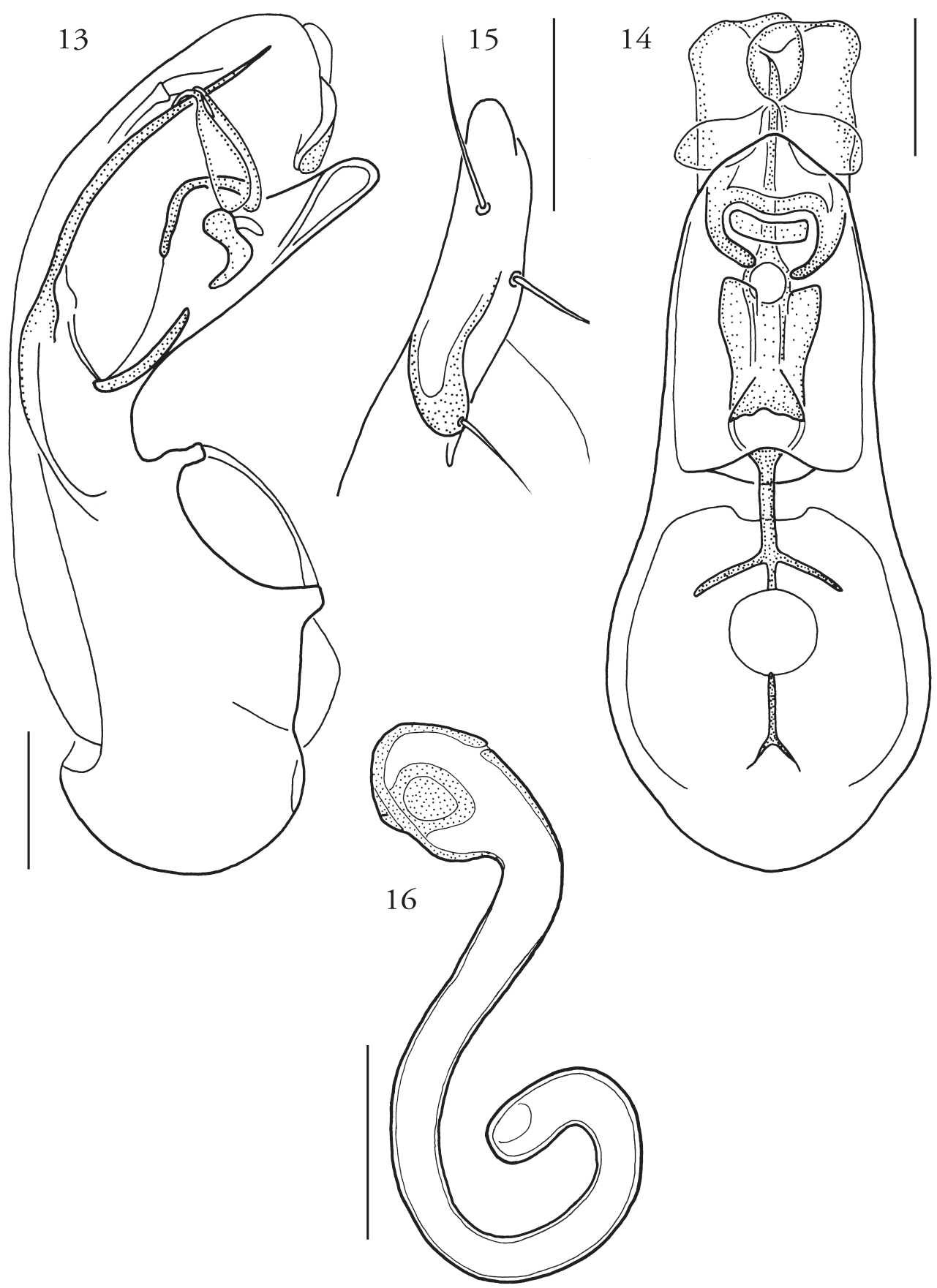

Fig. 13-16: Tectusa montana (KraAtz) [Maßstab 0,1 mm]. - 13 Aedoeagus, lateral [Lectotypus]. 14 Aedoeagus, ventral [Lectotypus]. 15 Apikalfortsatz der Paramere [Topotypus]. 16 Spermatheka [Topotypus]. 

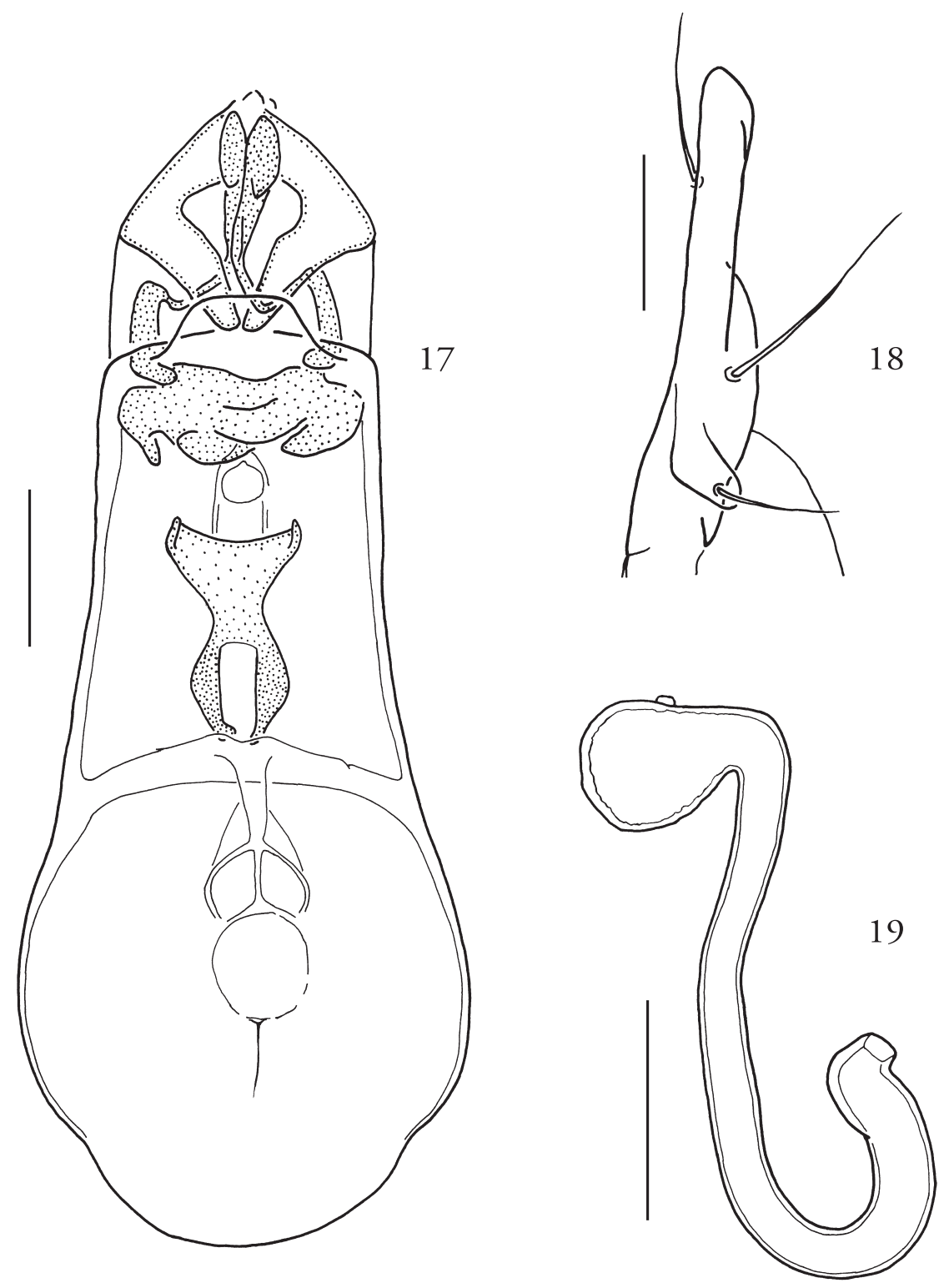

Fig. 17-19: Tectusa holdhausi Bernhauer [Maßstab 0,1 mm]. - 17 Aedoeagus, ventral [Topotypus]. 18 Apikalfortsatz der Paramere [Topotypus]. 19 Spermatheka [Holotypus]. 


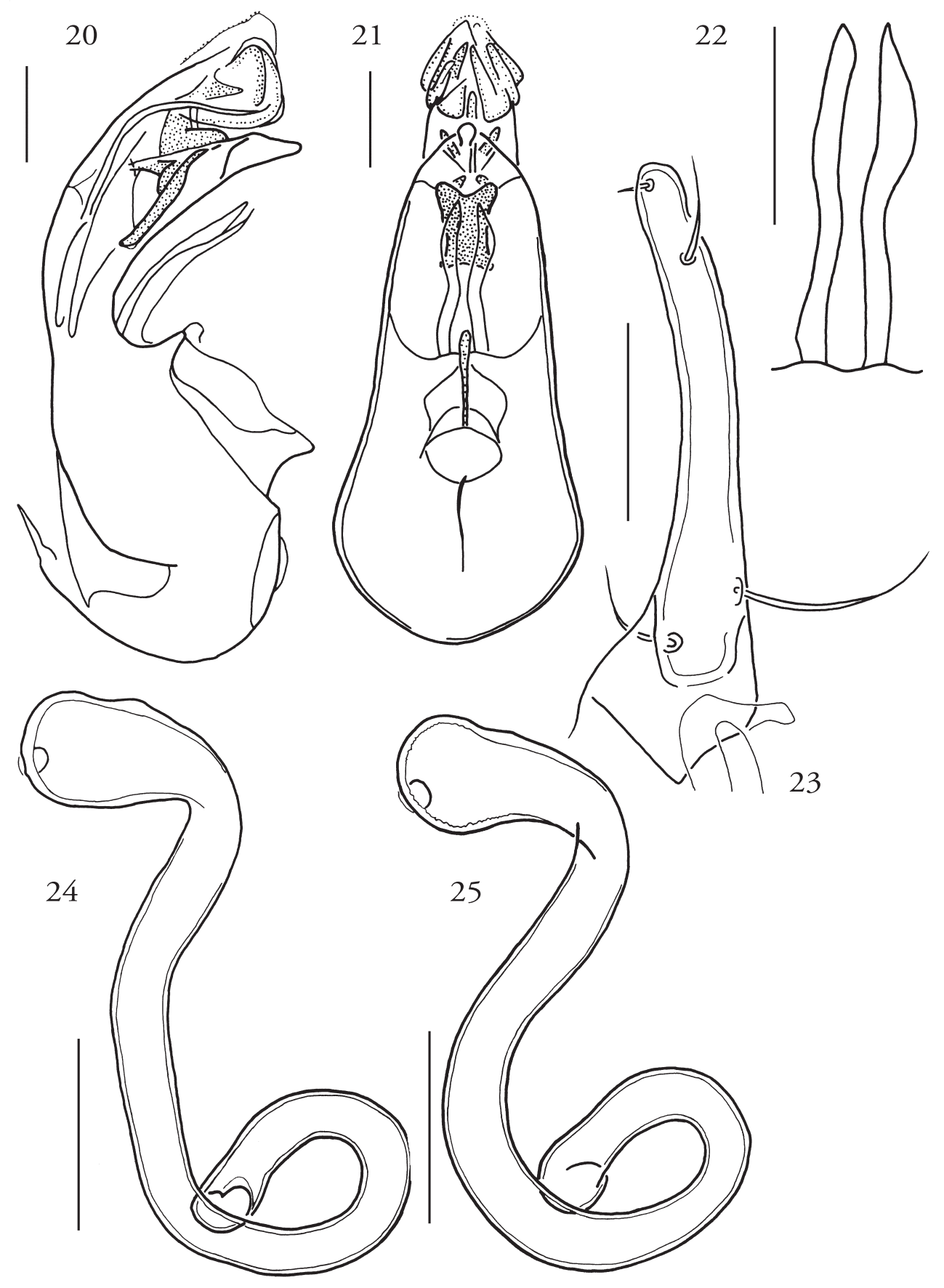

Fig. 20-25: Tectusa transsylvanica sp. n. - 20 Aedoeagus, lateral [Holotypus]. 21 Aedoeagus, ventral [Paratypus]. 22 Paarige ventrale Fortsätze des Aedoeagus [Paratypus]. 23 Apikalfortsatz der Paramere [Paratypus]. 24-25 Spermatheka [Paratypen]. 

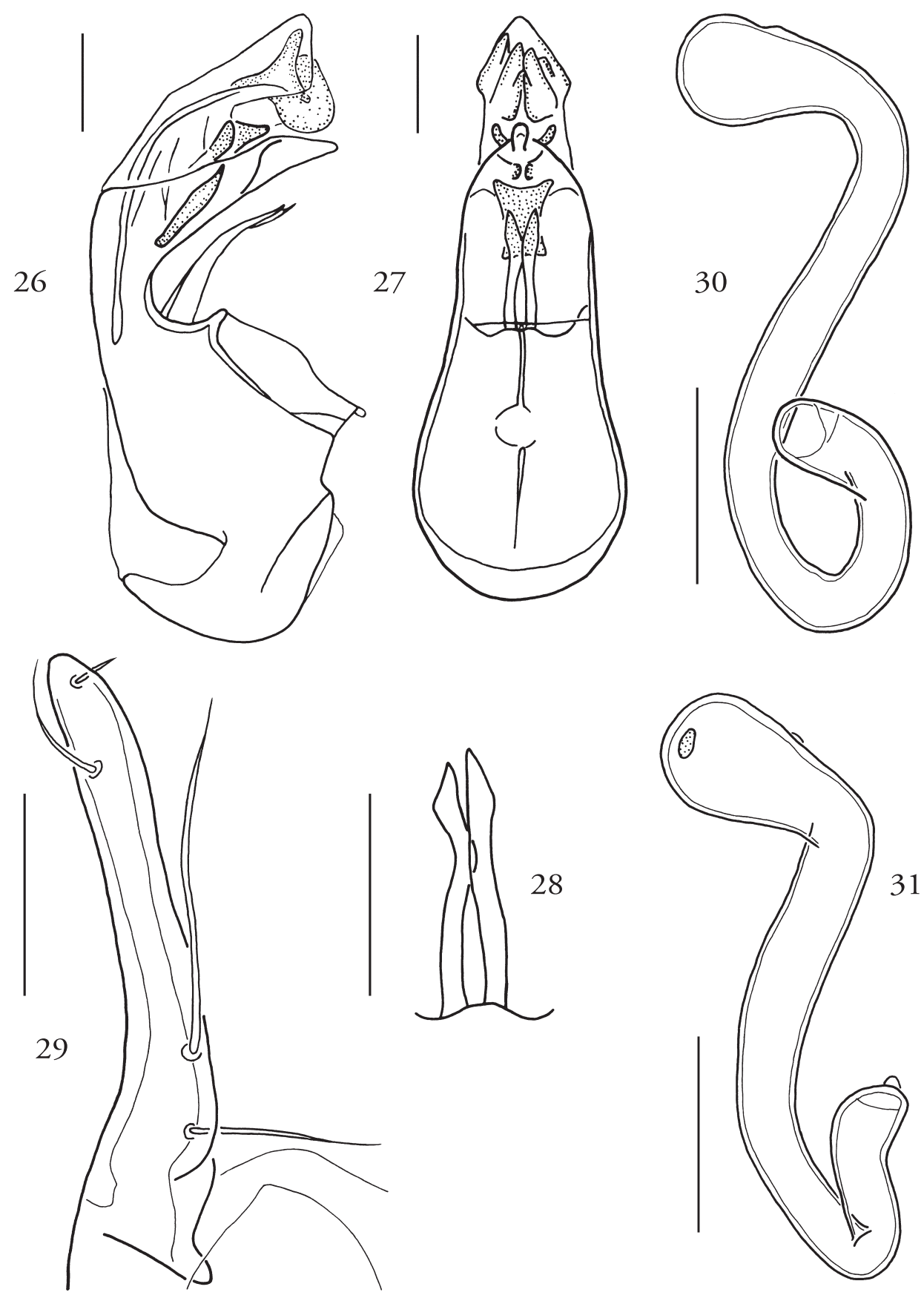

Fig. 26-31: Tectusa bucegiensis sp. n. - 26 Aedoeagus, lateral [Holotypus]. 27 Aedoeagus, ventral [Paratypus]. 28 Paarige ventrale Fortsätze des Aedoeagus [Paratypus]. 29 Apikalfortsatz der Paramere [Paratypus]. 30-31 Spermatheka [Paratypen]. 


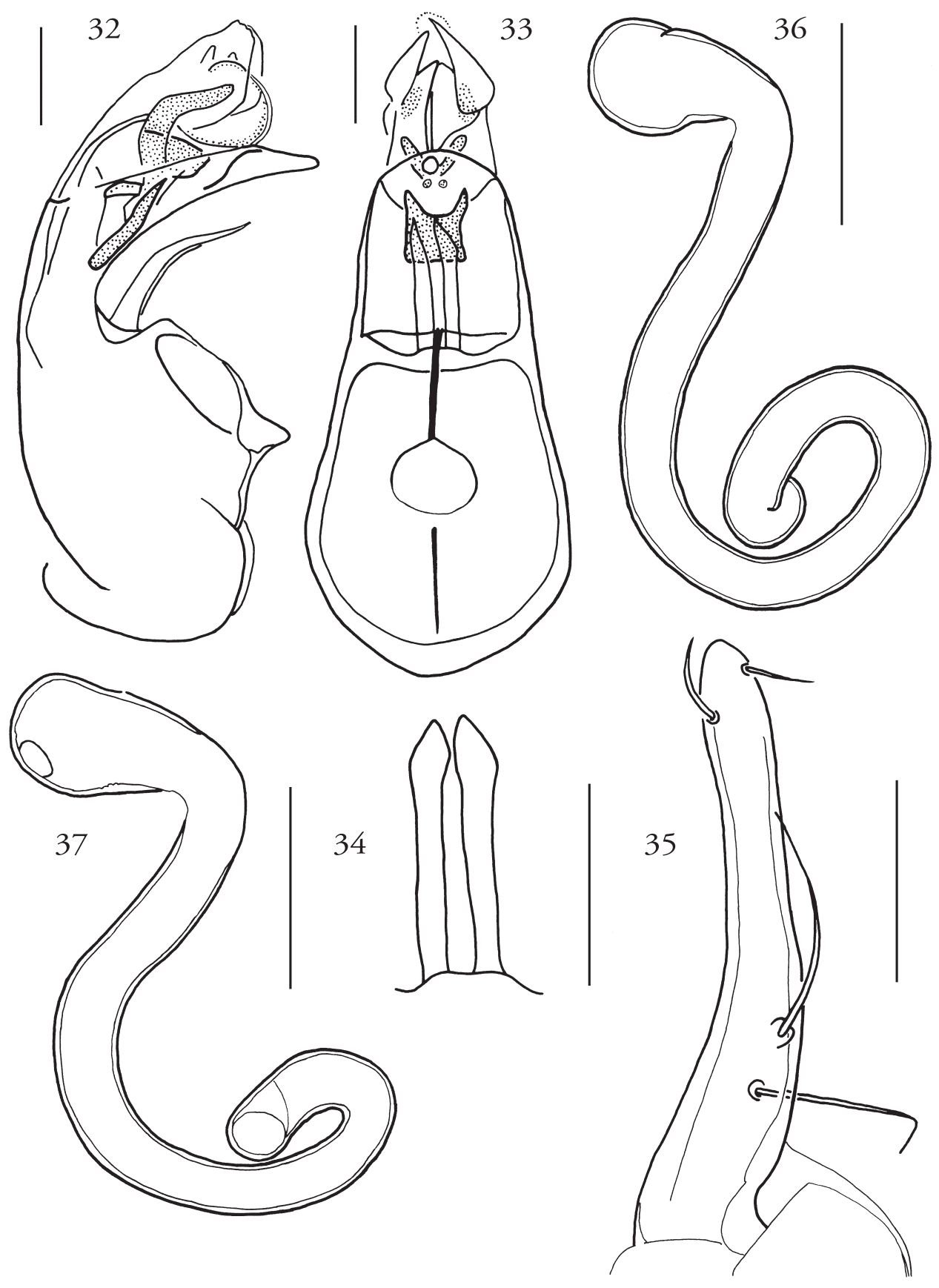

Fig. 32-37: Tectusa rosenauensis sp. n. - 32 Aedoeagus, lateral [Holotypus]. 33 Aedoeagus, ventral [Paratypus]. 34 Paarige ventrale Fortsätze des Aedoeagus [Paratypus]. 35 Apikalfortsatz der Paramere [Paratypus]. 36-37 Spermatheka [Paratypen]. 


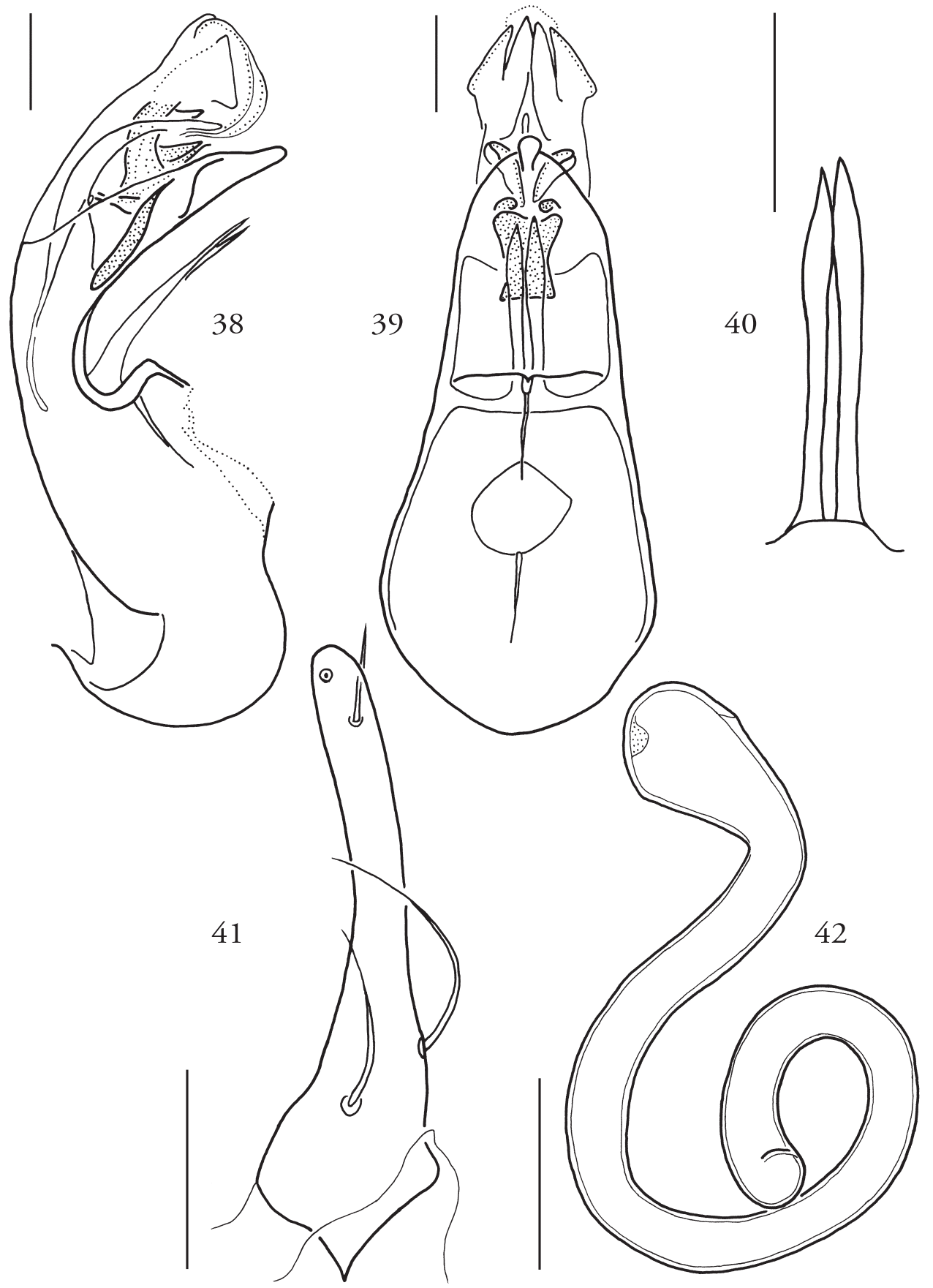

Fig. 38-41: Tectusa ceahlauensis sp. n. - 38 Aedoeagus, lateral [Holotypus]. 39 Aedoeagus, ventral [Holotypus]. 40 Paarige ventrale Fortsätze des Aedoeagus [Holotypus]. 41 Apikalfortsatz der Paramere [Holotypus]. Fig. 42 Tectusa spec. (Roter-Turm-Pass). - Spermatheka. 


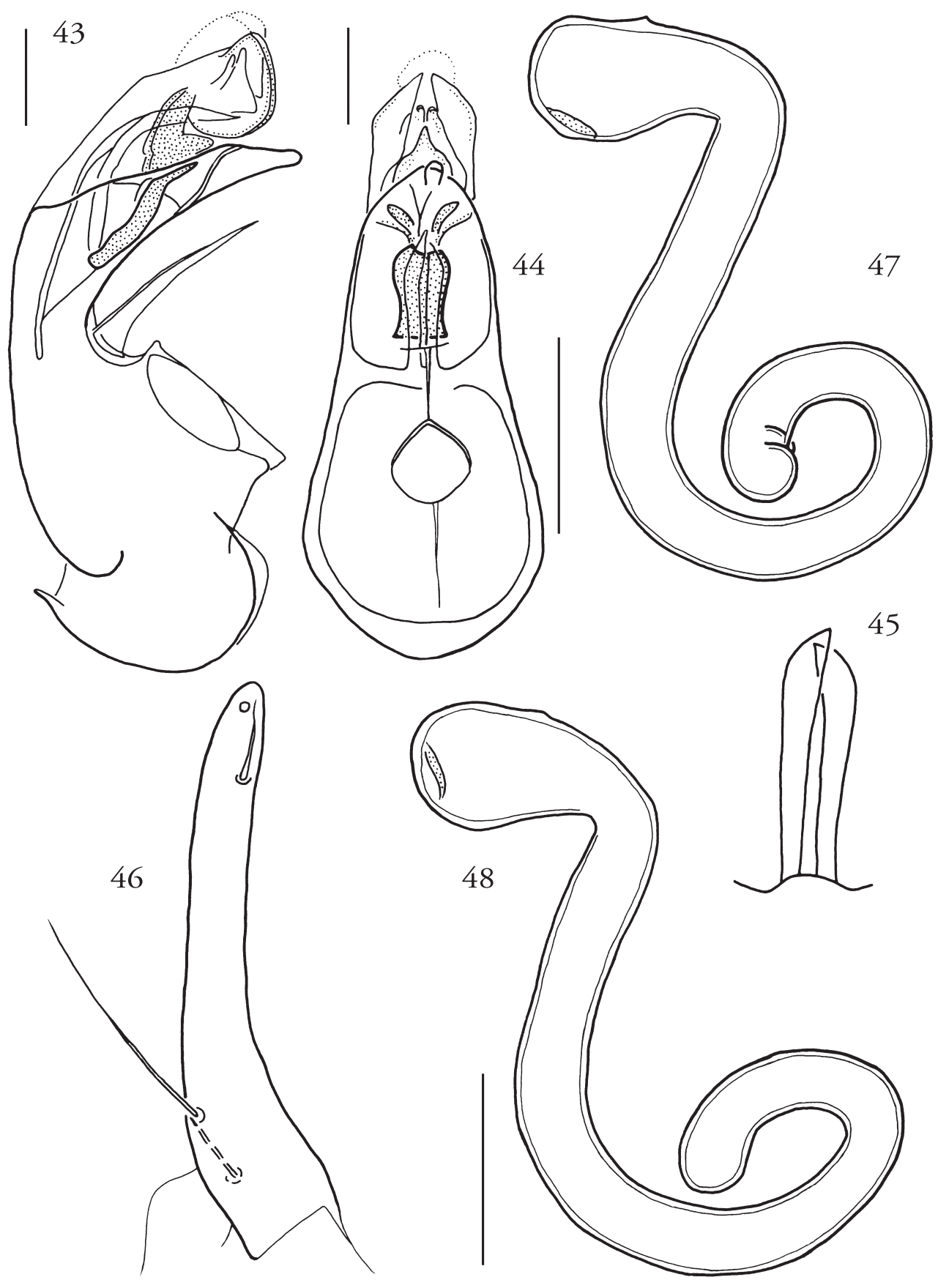

Fig. 43-48: Tectusa rodnaensis sp. n. - 43 Aedoeagus, lateral [Holotypus]. 44 Aedoeagus, ventral [Paratypus]. 45 Paarige ventrale Fortsätze des Aedoeagus [Paratypus]. 46 Apikalfortsatz der Paramere [Paratypus]. 47-48 Spermatheka [Paratypen]. 


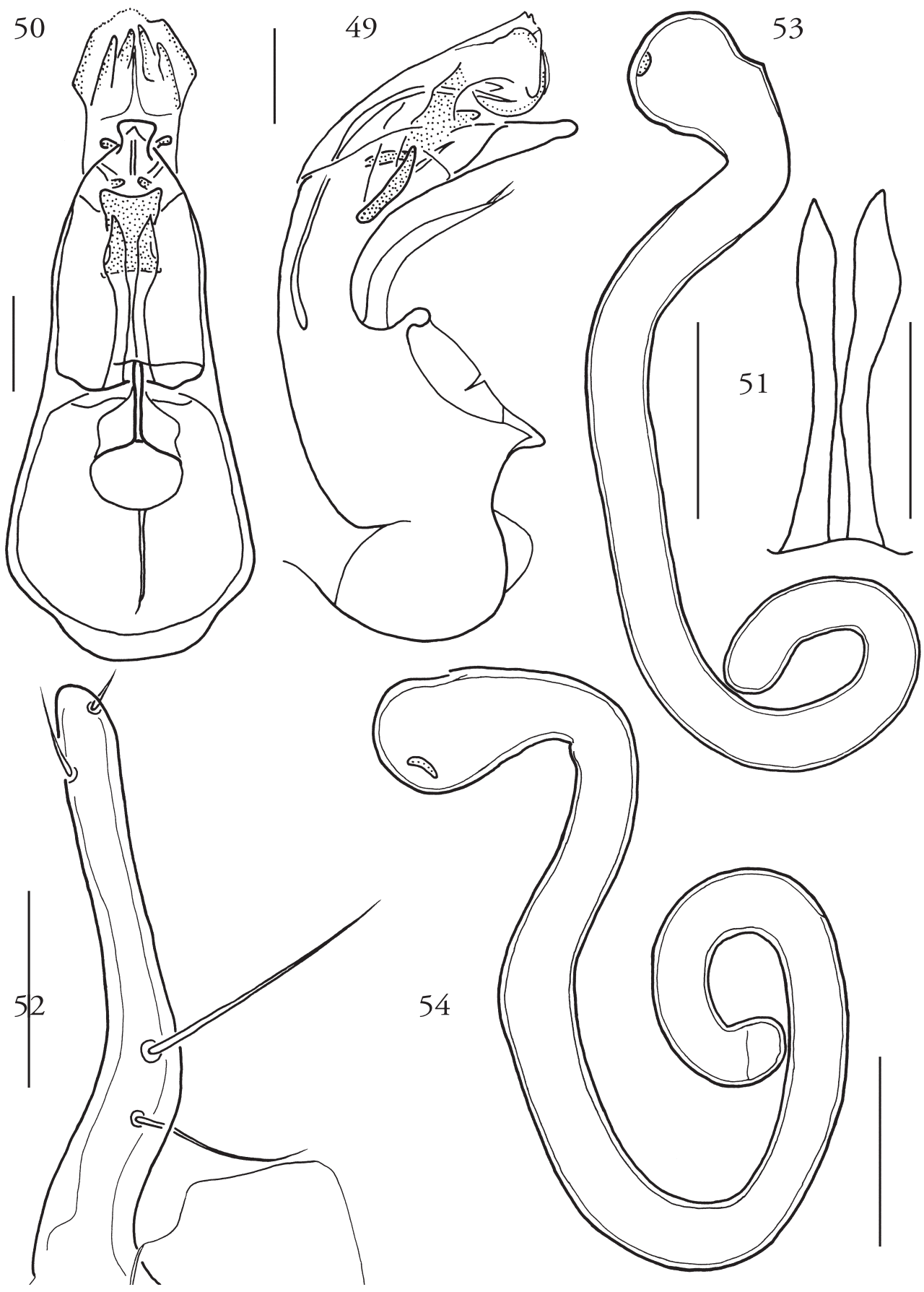

Fig. 49-54: Tectusa nigromontis sp. n. - 49 Aedoeagus, lateral [Holotypus]. 50 Aedoeagus, ventral [Paratypus]. 51 Paarige ventrale Fortsätze des Aedoeagus [Paratypus]. 52 Apikalfortsatz der Paramere [Paratypus]. 53-54 Spermatheka [Paratypen]. 